\title{
LncRNA SPRY4-IT1 promotes progression of osteosarcoma by regulating ZEB1 and ZEB2 expression through sponging of miR-101 activity
}

\author{
HUI YAO*, GANG HOU*, QI-YOU WANG, WEN-BIN XU, HUI-QING ZHAO and YI-CHUN XU \\ Department of Orthopaedics, The Third Affiliated Hospital of Sun Yat-Sen University, \\ Guangzhou, Guangdong 510530, P.R. China
}

Received March 25, 2019; Accepted September 16, 2019

DOI: $10.3892 /$ ijo.2019.4910

\begin{abstract}
Long non-coding (lnc)RNA sprouty receptor tyrosine kinase signalling antagonist 4-intronic transcript 1 (SPRY4-IT1) has been demonstrated to serve a critical role in the tumorigenesis of osteosarcoma (OS); however, the specific underlying mechanism remains unclear. The aim of the present study was to examine the interactions between SPRY4-IT1 and its downstream effectors, to determine if any of the interactions contributed to SPRY4-IT1-mediated proliferation, migration and invasion in cancer cells. A signalling cascade which involved SPRY4-IT1, miR-101 and zinc finger E-box-binding homeoboxes (ZEBs) was examined in the present study. Intracellular SPRY4-IT1 and miR-101 expression levels were altered through transfection to assess their effect on proliferation, cell cycle progression, survival, migration and invasion. A dual-luciferase assay was utilized to determine the association between SPRY4-IT1/miR-101 and ZEBs/miR-101 and nude mouse xenograft experiments were performed to determine the effect of SPRY4-IT1 in vivo. The results indicated that the SPRY4-IT1 levels were negatively associated with miR-101 expression levels in OS cells, an association which was not observed in the normal osteoblast cells. SPRY4-IT1 knockdown or miR-101 overexpression reduced proliferation, cell cycle progression, survival, migration and invasion of MG-63 and U2OS cells. SPRY4-IT1 knockdown was accompanied by increased expression of miR-101 and
\end{abstract}

Correspondence to: Dr Yi-Chun Xu or Dr Hui-Qing Zhao, Department of Orthopaedics, The Third Affiliated Hospital of Sun Yat-Sen University, 2693 Kaichuang Road, Guangzhou, Guangdong 510530, P.R. China

E-mail: xuyichun808@163.com

E-mail: zhaohuiqing668@163.com

*Contributed equally

Key words: sprouty receptor tyrosine kinase signalling antagonist 4intronic transcript 1, microRNA-101, zinc finger E-box-binding homeoboxes, osteosarcoma
E-cadherin levels, as well as decreased expression levels of ZEB1/2 and other epithelial-mesenchymal transition-associated proteins. Simultaneous knockdown of SPRY4-IT1 and inhibition of miR-101 partially reversed the anti-tumour effects of SPRY4-IT1 inhibition in vitro. Consistent with these findings, short hairpin RNA targeting SPRY4-IT1 also hindered xenograft tumour growth and altered the levels of miR-101, ZEB1/2 and E-cadherin in vivo. Dual-luciferase reporter assays demonstrated that SPRY4-IT1 may have regulated the expression of ZEB1 and ZEB2 by sponging miR-101. In conclusion, SPRY4-IT1 inhibition increased miR-101 levels, resulting in downregulation of ZEB1/2 expression and thus exerting anti-tumour effects in OS.

\section{Introduction}

It has been reported that over $80 \%$ of osteosarcoma (OS) patients would die of metastases if they did not receive chemotherapy (1). Although multimodal treatment approaches have improved overall survival, survival rates are still far from satisfactory. Currently, the 5-year overall survival rate of patients with OS is $\sim 60 \%$, even if patients received intensive chemotherapy (2). Therefore, uncovering the underlying mechanisms of tumorigenesis of OS may assist in the development of improved treatments and targeting genes that promote progression of OS may hold potential for improving the efficiency of treatments in the future.

Epithelial-mesenchymal transition (EMT) is crucial for progression of OS (3). Whilst effectors of EMT, such as E-cadherin, N-cadherin, vimentin, fibronectin, matrix metalloproteinase (MMP)-2 and MMP-9 have been extensively studied (4), the identities of the upstream modulators of these markers are less clear. Zinc finger E-box-binding homeobox (ZEB) family members are candidates for the regulation of EMT (3). In OS, increased expression of ZEB1 and ZEB2 has been associated with cancer development and poor prognosis (5). Increased expression levels of ZEB1 transcriptionally represses the expression of E-cadherin (6), which in turn, favours migration of cancer cells (3). Furthermore, by interacting with the Wnt pathway or p73 proteins, ZEBs can also promote cell cycle progression and cell survival in different types of cancer, including colon, lung and pancreatic 
cancer (7). In this regard, investigating negative regulators of ZEB family members may provide additional opportunities for preventing or reversing EMT and serve as an alternative anti-tumour therapy.

Emerging evidence has shown that non-coding RNAs including long non-coding RNAs (lncRNAs; RNA transcripts $>200$ bp in length) and microRNAs (miRNAs; RNA transcripts $\sim 22$ bp in length) are intrinsically involved in regulation of protein expression at all levels of the protein production process $(8,9)$. Transcriptome studies have demonstrated multiple series of dysregulated miRNAs in various tumours, including OS $(10,11)$. miRNAs directly bind to protein-coding mRNAs and prevent them from being translated into proteins, and during the pathogenesis of OS this mechanism is used to suppress expression tumour suppressor genes (12). In the field of lncRNAs, the mechanisms of regulation of expression of genes is more complicated and diverse. Through binding to chromatin, mRNAs or proteins, lncRNAs can decrease or increase the expression of protein-coding genes and have been reported to be involved in almost all aspects of OS (13). Previous studies on the crosstalk between lncRNAs and miRNAs have revealed a new mechanism of protein-coding gene modulation (14-16). LncRNAs can act as competing endogenous RNAs (ceRNAs) and de-repress (increase) the expression of protein-coding genes by sequestering miRNAs (17). CeRNA-dependent regulation, therefore, represents a novel mechanism for the upregulation of oncogenes.

Sprouty receptor tyrosine kinase signalling antagonist 4-intronic transcript 1 (SPRY4-IT1) is a lncRNA that has been associated with several types of cancer, including OS (18). Overexpression of SPRY4-IT1 in OS cells promoted proliferation, survival and migration; however, the downstream effectors of SPRY4-IT1 remain unknown (19). As ZEBs promote tumour development, it was hypothesized that SPRY4-IT1 may function by regulating ZEB expression or function. miR-101, a tumour suppressor gene, was reported to suppress EMT by targeting ZEB1 and ZEB2 in ovarian carcinoma (20). Furthermore, SPRY4-IT1 has been shown to promote the proliferation and migration of bladder cancer cells by sponging miR-101 (21). However, the association between SPRY4-IT1 and miR-101 and the functional interaction between miR-101 and ZEB1/ZEB2 have not been explored in OS. The hypothesis of the present study was that SPRY4-IT1 acted as a ceRNA to sequester miR-101, resulting in an increase in the levels of ZEB1/2 proteins, which in turn promoted the proliferation, migration and invasion of OS cells.

In the present study, the role of SPRY4-IT1 in the tumorigenesis of OS was investigated. The aim of the present study was to explore a miR-101 and ZEB1/ZEB2 axis as a potential downstream effector of SPRY4-IT1 involved in proliferation, survival, migration and invasion in OS. The present study may provide insights into a novel strategy for treatment of OS.

\section{Materials and methods}

Cell culture. The human normal osteoblast cell line hFOB 1.19 (ATCC ${ }^{\circledR}$ CRL-11372 ${ }^{\mathrm{TM}}$ ), the OS cell lines U2OS (ATCC ${ }^{\circledR}$ HTB-96 ${ }^{\mathrm{TM}}$ ), MG-63 (ATCC ${ }^{\circledR}$ CRL-1427 ${ }^{\mathrm{TM}}$ ) and Saos-2 cells (ATCC ${ }^{\circledR}$ HTB-85 $^{\mathrm{TM}}$ ), and normal human 293 cells $\left(\right.$ ATCC $^{\circledR}$
CRL-1573 ${ }^{\mathrm{TM}}$ ) were obtained from ATCC. All cells were maintained in DMEM (Gibco; Thermo Fisher Scientific, Inc.) supplemented with $10 \%$ heat-inactivated FBS (Gibco; Thermo Fisher Scientific, Inc.) and 1\% penicillin-streptomycin solution (Sigma-Aldrich; Merck KGaA) in a humidified incubator with $5 \% \mathrm{CO}_{2}$ at $37^{\circ} \mathrm{C}$.

Transfection. miRNA mimics, inhibitor, scrambled control oligos, pGPH1 plasmid containing short hairpin (sh)RNA targeting SPRY4-IT1 and pGPH1 plasmid containing scrambled control shRNA were purchased from Shanghai GenePharma Co., Ltd. For transfection of miRNAs, MG-63 or U2OS cells were seeded into 6-well plates and cultured with supplemented media overnight. When the confluency of cells reached $\sim 80 \%$, the medium was replaced with DMEM without serum. A total of 25 pmol miR-101 mimics/inhibitor or its scrambled control (NC) was mixed with $7.5 \mu 1$ Lipofectamine ${ }^{\circledR}$ RNAiMAX reagent (Thermo Fisher Scientific, Inc.) and added to the cells. A total of $6 \mathrm{~h}$ later, the medium containing the oligos for transfection and Lipofectamine ${ }^{\circledR}$ was removed and replaced with supplemented DMEM without antibiotics. RNA or protein lysates were collected 24 or $48 \mathrm{~h}$ after transfection, respectively. For RNA interference studies, $1 \mathrm{mg}$ pGPH1 plasmid and $3 \mathrm{ml}$ Lipofectamine $^{\circledR} 2000$ (Thermo Fisher Scientific, Inc.) were mixed and added to MG-63 or U2OS cells. Cells were cultured with the transfection reagent and plasmid for $48 \mathrm{~h}$ before subsequent assays were performed unless otherwise stated.

Cell proliferation assays. Cell proliferation was evaluated using MTT and colony formation assays. For the MTT assay, MG-63 or U2OS cells were seeded into 96-well plates at a density of $5 \times 10^{3}$ cells/well and transfected with the miR-101 mimics, inhibitor or shSPRY4-IT1 for 24, 48, 72 or $96 \mathrm{~h}$. After the indicated amount of time, $10 \mu \mathrm{l}$ MTT solution (Sigma-Aldrich; Merck KGaA) in PBS (5 mg/ml) was added to each well and incubated in a cell culture incubator for $3 \mathrm{~h}$. The supernatant was removed carefully, and the formazan crystals were dissolved in $100 \mu$ l DMSO (Sigma-Aldrich; Merck KGaA). The cell viability in each well was determined by measuring the optical density at $490 \mathrm{~nm}$.

For the colony formation assay, MG-63 or U2OS cells were seeded and transfected with miRNA mimics, inhibitors or shSPRY4-IT1. Cells were then re-seeded in 6-well plates at a density of 500 cells/well and allowed to grow for an additional 15 days. Cells were then fixed with $100 \%$ methanol for $10 \mathrm{~min}$ at $-20^{\circ} \mathrm{C}$ and stained with $0.5 \%$ crystal violet (in $25 \%$ methanol) for $10 \mathrm{~min}$ at room temperature. The colonies were counted by two researchers who were blinded to the experimental conditions.

Cell apoptosis analysis. MG-63 or U2OS cells were seeded in 12 -well plates at a density of $1 \times 10^{5}$ cells/well. After transfection, the cells were trypsinized and harvested for staining using an Annexin V-fluorescein isothiocyanate (FITC)/propidium iodide (PI) Detection kit (Sigma-Aldrich; Merck KGaA) according to the manufacturer's protocol. Cells were analysed by flow cytometry (Becton, Dickinson and company). The $\mathrm{FITC}^{+} / \mathrm{PI}^{-}$and $\mathrm{FITC}^{+} / \mathrm{PI}^{+}$fractions were considered early and late apoptotic cells, respectively. Analysis of flow data was performed using FlowJo version X.10.0.7-1 (FlowJo LLC). 
Assessment of cell cycle progression. Cell cycle progression was evaluated using flow cytometry. For flow cytometry, after transfection as described above, MG-63 or U2OS cells were trypsinized and harvested and fixed with ice-cold $70 \%$ ethanol overnight at $-20^{\circ} \mathrm{C}$. Subsequently, the cells were incubated with PBS solution containing PI $(50 \mu \mathrm{g} / \mathrm{ml})$ and RNase A $(30 \mu \mathrm{g} / \mathrm{ml})$ for $15 \mathrm{~min}$ at $37^{\circ} \mathrm{C}$. Cell cycle distribution was analysed by flow cytometry (Becton, Dickinson and Company).

Cell migration and invasion assays. Cell migration and invasion were evaluated using a wound healing assay and Transwell invasion assay, respectively. For the wound healing assay, MG-63 or U2OS cells were seeded onto 12-well plates and transfected as described above. After $48 \mathrm{~h}$ of transfection, a wound was created by scratching the cell monolayer with a $1 \mathrm{ml}$ pipette tip. Cells were then washed with PBS three times and incubated for an additional $24 \mathrm{~h}$ in serum-free culture medium. Distances were measured and analysed by ImageJ software (NIH, US). Relative wound closure was measured using the following formula: $\left(\mathrm{W}_{0}\right.$ $\left.{ }_{\mathrm{h}}-\mathrm{W}_{24 \mathrm{~h}}\right) / \mathrm{W}_{0 \mathrm{~h}} \times 100 \%$; where $\mathrm{W}$ is the width.

Matrigel-coated Transwell inserts or uncoated inserts with $5-\mu \mathrm{m}$ pores were used (Corning, Corning, NY, USA) in a 24 well plate for Transwell invasion and migration assays, respectively. MG-63 or U2OS cells were transfected as described above and subsequently $5 \times 10^{4}$ cells were added to the top chamber of the inserts with $400 \mu \mathrm{l}$ serum-free culture medium. The bottom compartments were filled with DMEM containing 20\% FBS as a chemoattractant. After $24 \mathrm{~h}$ of culture, the inserts were collected, the non-invading or non-migrating cells on the upper surface were removed with a cotton swab, and the invaded or migrated cells on the lower surface were fixed with $4 \%$ paraformaldehyde for $10 \mathrm{~min}$ at room temperature and stained with crystal violet for $10 \mathrm{~min}$ at room temperature according to manufacturer's protocol (Sigma-Aldrich; Merck KGaA). The invaded or migrated cells were counted under an inverted light microscope with a x100 magnification (Carl Zeiss AG) by researchers who were blinded to the experimental conditions.

Dual-luciferase reporter assay. The predicted miR-101 binding sites on SPRY4-IT1 were predicted using RNAInter website (rna-society.org/raid/search.html). The predicted miR-101 binding sites on ZEB1 or ZEB2 were predicted using starBase (starbase.sysu.edu.cn/index.php). Dual-luciferase reporter assays were performed to determine whether miR-101 was targeted by SPRY4-IT1 and the 3' untranslated region (UTR) ZEB1/2. To confirm the interaction between miR-101 and SPRY4-IT1, SPRY4-IT1 gene fragments containing a predicted miR-101 binding site or corresponding mutant fragments were cloned into pGL3-basic vectors (Promega Corporation). miR-101 mimics or inhibitor were used for miR-101 overexpression or knockdown, respectively. A pRL Renilla Luciferase vector (Promega Corporation) was used as the control plasmid. During transfection, miR-101 mimics or inhibitor and pGL3-basic vectors were co-transfected into 293T cells with Lipofectamine ${ }^{\circledR} 2000$ (Thermo Fisher Scientific, San Jose, CA, USA). Scrambled oligos were used as the control. After transfection for $48 \mathrm{~h}$, firefly luciferase activity was determined and adjusted based on the Renilla luminescence activity according to the manufacturer's protocol (Promega Corporation).
For the 3'UTR assay, oligos containing putative miR-101 binding sites were cloned from the ZEB1 or ZEB2 3'UTR and inserted into pmir-GLO vectors (Promega Corporation). The ZEB1 and ZEB2 3'UTR, with the predicted microRNA seed binding regions mutated were generated through site-directed mutagenesis kit (Promega Corporation). The primer sequence for mutation of ZEB1 3'UTR was 5'-TAAAAATGTTGC ACAG-3'. The primer sequence for mutation of ZEB2 3'UTR was 5'-CTTGAAATAAATAAAAT-3'. During transfection, miR-101 mimics or inhibitor and pmir-GLO vectors were co-transfected into $293 \mathrm{~T}$ cells with Lipofectamine ${ }^{\circledR} 2000$. After transfection for $48 \mathrm{~h}$, relative luciferase activity was measured.

Establishment of stable cell lines. MG-63 or U2OS cells were transfected with shNC (scramble control) or shSPRY4-IT1 plasmids. Transfected cells were selected for using $400 \mathrm{mg} / \mathrm{ml}$ Geneticin (Thermo Fisher Scientific, Inc.). Stably transfected cells were maintained in culture medium containing $100 \mathrm{mg} / \mathrm{ml}$ Geneticin and then used in the nude mouse xenograft study.

Xenograft tumour model. BALB/c nude mice were purchased from Shanghai SLAC Laboratory Animal Center (Shanghai, China). The nude mouse study was approved by the Animal Ethics Committee at the Third Affiliated Hospital of Sun Yat-sen University (Guangzhou, China). A total of 24 nude mice were randomly assigned to 4 groups ( $n=6 /$ group); MG-63/shNC (control), MG-63/shSPRY4-IT1 (treatment), $\mathrm{U} 2 \mathrm{OS} / \mathrm{shNC}$ (control) and U2OS/shSPRY4-IT1 (treatment). A total of $1 \times 10^{6}$ cells $/ 100 \mathrm{ml} \mathrm{MG}-63$ or U2OS cells, which were stably transfected with shNC or shSPRY4-IT1, were inoculated subcutaneously in the nude mice. The xenograft tumour size was measured every 5 days using a Vernier scale. After 30 days, the mice were sacrificed by cervical dislocation. The tumour tissues were harvested for volume and weight measurement, RT-qPCR and western blot analysis.

$R N A$ isolation and reverse transcription-quantitative (RT-q) $P C R$. Total RNA from xenograft tumours and cells was extracted using TRIzol ${ }^{\circledR}$ reagent (Thermo Fisher Scientific, Inc.) according to the manufacturer's protocol. Briefly, tumours or cells were dissolved in $1 \mathrm{ml} \mathrm{TRIzol}{ }^{\circledR}$, followed by total RNA extraction with $200 \mu \mathrm{l}$ chloroform and RNA precipitation with $500 \mu \mathrm{l}$ isopropanol. cDNA was synthesized from $1 \mu \mathrm{g}$ total RNA using the PrimeScript RT reagent kit (Takara Bio, Inc.). Reverse transcription as performed as follows: $25^{\circ} \mathrm{C}$ for $5 \mathrm{~min}$, $37^{\circ} \mathrm{C}$ for $30 \mathrm{~min}$ and $85^{\circ} \mathrm{C}$ for $5 \mathrm{sec}$. cDNA was diluted 20 -fold with $\mathrm{ddH}_{2} \mathrm{O}$ and used for qPCR with SYBR Premix EX Taq kit (Takara Bio, Inc.) in an ABI 7500HT real-time PCR system (Thermo Fisher Scientific, Inc.). The thermocycling conditions were as follows: Initial denaturation, $95^{\circ} \mathrm{C}$ for $5 \mathrm{sec}$; followed by 35 cycles of denaturation at $94^{\circ} \mathrm{C}$ for $15 \mathrm{sec}$, annealing at $55^{\circ} \mathrm{C}$ for $25 \mathrm{sec}$ and extension at $70^{\circ} \mathrm{C}$ for $30 \mathrm{sec}$. The gene levels for all samples were normalized to U6 small nuclear (sn)RNA (for miRNA) or GAPDH levels using the $2^{-\Delta \Delta C q}$ method (22).

Western blotting. Total protein was extracted with cell lysis buffer (50 mM Tris, $150 \mathrm{mM} \mathrm{NaCl}, 1 \%$ NP-40, 1 mM EDTA, $\mathrm{pH}$ 7.6) containing a cocktail of protease inhibitors (Thermo 
Fisher Scientific, Inc.). Protein concentration was determined using a bicinchoninic acid assay kit (Pierce; thermo Fisher Scientific, Inc.) according to the manufacturer's protocol. Samples (30 $\mu \mathrm{g}$ protein/lane) were loaded on a $10 \%$ SDS gel and resolved using SDS-PAGE and then transferred onto PVDF membranes $(0.22 \mu \mathrm{m}$ pore, Roche). After blocking with TBS-Tween buffer (20 mM Tris, $137 \mathrm{mM} \mathrm{NaCl}, 0.1 \%$ Tween-20, $\mathrm{pH} 8.0$ ) containing 5\% non-fat milk for $1 \mathrm{~h}$ at room temperature, the membranes were incubated with primary antibodies against ZEB1 (cat. no. 3396), ZEB2 (cat. no. 97885), E-cadherin (cat. no. 3195), Vimentin (cat. no. 5741), Fibronectin (cat. no. ab2413), N-cadherin (cat. no. 13116), MMP-2 (cat. no. 40994), MMP-9 (cat. no. 13667) or GAPDH (cat. no. 5174) overnight at $4^{\circ} \mathrm{C}$. All primary antibodies were purchased from Cell Signaling Technology, Inc. except for Fibronectin, which was purchased from Abcam, and all antibodies were used at a dilution of 1:1,000. Subsequently, membranes were incubated with a goat anti-rabbit (\#7074) horseradish peroxidase-conjugated secondary antibody (1:3,000; Cell Signaling Technology, Inc.) for $1 \mathrm{~h}$ at room temperature. The protein bands were visualized using Immobilon Western Chemiluminescent horseradish peroxidase substrate (EMD Millipore). The proteins were quantified using Quantity One version 4.2.1 (Bio-Rad Laboratories, Inc.).

Statistical analysis. All experiments were performed at least three times in triplicate. Data are presented as the mean \pm standard deviation. All statistical analyses were performed in GraphPad Prism version 6 (GraphPad Software, Inc.). Statistical evaluation was performed using a two-tailed Student's t-test between two groups or a one-way ANOVA followed by a post hoc Tukey's test for multiple comparisons. $\mathrm{P}<0.05$ was considered to indicate a statistically significant difference.

\section{Results}

SPRY4-IT1 knockdown results in increased miR-101 expression in OS cells. The expression of SPRY4-IT1 and miR-101 was detected in three OS cell lines, Saos-2, MG-63 and U2OS, as well as the normal osteoblast cell line hFOB 1.19. Compared with the hFOB 1.19 cells, SPRY4-IT1 was significantly upregulated in all three OS cell lines (Fig. 1A). In contrast, a reduction in miR-101 was observed in the three tumour cell lines (Fig. 1B), highlighting a potential functional interaction between SPRY4-IT1 and miR-101. To examine this potential interaction, SPRY4-IT1 was knocked down in MG-63 and U2OS cells. As shown in Fig. 1C, shSPRY4-IT1 transfection significantly decreased the expression of SPRY4-IT1 compared with the control and shNC groups. This was accompanied by a significant increase in miR-101 expression (Fig. 1D). However, SPRY4-IT1 expression were not altered in cells transfected with miR-101 (Fig. 1E and F). These results suggest a unidirectional crosstalk between SPRY4-IT1 and miR-101, such that SPRY4-IT1 may function as an upstream modulator of miR-101 in OS cells.

SPRY4-IT1 silencing and miR-101 overexpression inhibits growth of OS cells. shSPRY4-IT1 or miR-101 mimic transfection was used to study the molecular functions of SPRY4-IT1 and miR-101, respectively. MTT and colony formation assays were first performed to investigate the effects of SPRY4-IT1 and miR-101 on cell growth. When compared with the scrambled control group, knockdown of SPRY4-IT1 or overexpression of miR-101 significantly reduced the growth of both MG-63 and U2OS cells in a time-dependent manner (Fig. 2A). Consistent with these results, colony formation assays revealed that SPRY4-IT1 knockdown or miR-101 overexpression both significantly reduced the number of colonies in MG-63 and U2OS cells (Fig. 2B and C). Furthermore, as shown in Fig. 2D and E, shSPRY4-IT1 transfection resulted in a significant increase in the apoptotic ratio of MG-63 (shSPRY4-IT1, 30.51\%; shNC, 8.56\%) and U2OS (shSPRY4-IT1, 24.61\%; shNC, $7.39 \%$ ) cells (both $\mathrm{P}<0.01$ ). Similarly, overexpression of miR-101 also greatly induced apoptosis in MG-63 (miR-101 mimics, 26.52\%; miR-101 NC, 6.96\%; $\mathrm{P}<0.05)$ and $\mathrm{U} 2 \mathrm{OS}$ (miR-101 mimics, 31.43\%; miR-NC 7.65\%; P<0.01) cells. The effects of SPRY4-IT1 knockdown or miR-101 overexpression on cell cycle progression of OS cells was assessed. shSPRY4-IT1 treatment of MG-63 and U2OS cells resulted in a significant accumulation of cells in the G1 phase, suggesting G1 phase arrest by SPRY4-IT1 knockdown (Fig. 2F and G). miR-101 mimic transfection resulted in $\mathrm{S}$ phase cell cycle arrest (Fig. 2F and G). Together, these results demonstrate that SPRY4-IT1 and miR-101 are involved in the growth of OS cells at least partially by modulating proliferation, apoptosis and cell cycle progression.

SPRY4-IT1 downregulation or miR-101 overexpression attenuates migration and invasion of OS cells. Increased cell migration and invasion have been identified as features of cancer metastasis, which is one of the hallmarks of cancer (23). The oncogenic function of SPRY4-IT1 and tumour suppressor function of miR-101 on cell migration and invasion were further explored. After $24 \mathrm{~h}$, SPRY4-IT1 downregulation or miR-101 induction significantly inhibited wound closure in both MG-63 and U2OS cells, suggesting that the migratory ability was reduced (Fig. 3A and B). A Transwell assay was also performed to evaluate the effect of SPRY4-IT1 and miR-101 on the migratory and invasive capacities of OS cells. shSPRY4-IT1 transfection significantly reduced the number of migrated or invaded MG-63 and U2OS cells (Fig. 3C-F). Similarly, overexpression of miR-101 also significantly reduced the migration or invasion of MG-63 and U2OS cells (Fig. 3C-F). Taken together, our results suggested that SPRY4-IT1 promoted cell migration and invasion, whereas miR-101 functioned as a negative regulator of these properties in OS cells.

SPRY4-IT1 and miR-101 modulate the expression of ZEB1 and ZEB2 in OS cells. Previously, it was reported that miR-101 may regulate the expression of both ZEB1 and ZEB2, both of which have been demonstrated to serve important roles in invasion and metastasis of lung and ovarian carcinoma $(20,24)$. Therefore, the effects of SPRY4-IT1 and miR-101 on regulation of ZEB1 and ZEB2 expression were determined. qPCR analysis showed that transfection of shSPRY4-IT1 or miR-101 mimics was sufficient to significantly reduce the mRNA expression levels of ZEB1 and ZEB2 in both MG-63 and U2OS cells (Fig. 4A-D). Western blotting also showed that SPRY4-IT1 knockdown and miR-101 overexpression 

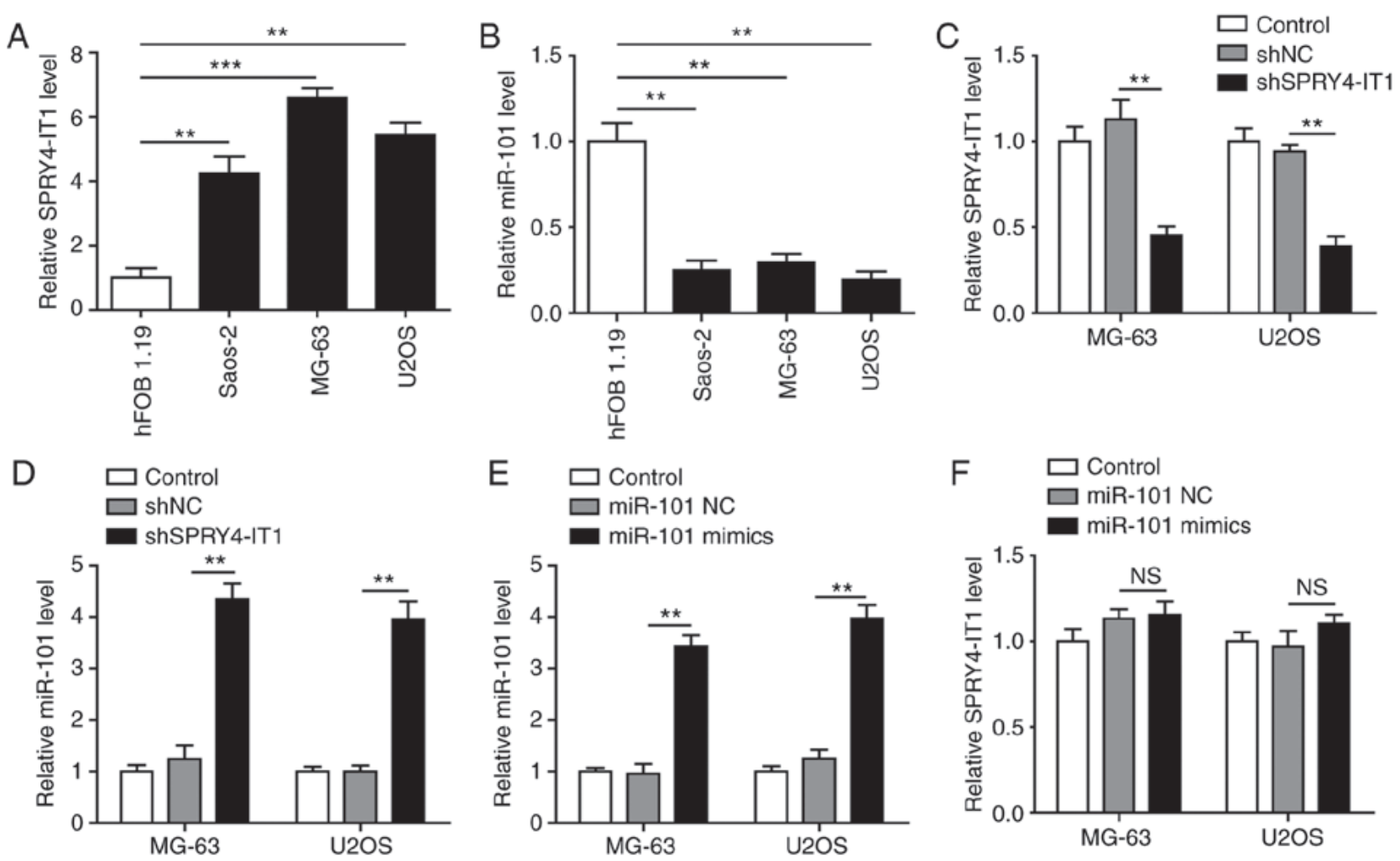

Figure 1. Increased SPRY4-IT1 expression results in reduced miR-101 expression in OS cells. (A) Expression of SPRY4-IT1 was significantly upregulated in OS cells compared with the normal osteoblast cells. (B) Expression of miR-101 was significantly downregulated in OS cells compared with normal osteoblast cells. (C) Transfection of shSPRY4-IT1 significantly reduced SPRY4-IT1 levels in MG-63 and U2OS cells. (D) SPRY4-IT1 knockdown resulted in upregulation of miR-101 expression levels in OS cells. (E) Transfection with miR-101 mimics significantly increased miR-101 expression levels. (F) Transfection of miR-101 mimics did not affect SPRY4-IT1 expression. Data are presented as the mean \pm standard deviation of three independent experiments. ${ }^{* *} \mathrm{P}<0.01,{ }^{* * *} \mathrm{P}<0.001$. n.s. not significant; SPRY4-IT1, sprouty receptor tyrosine kinase signalling antagonist 4-intronic transcript 1; miR, microRNA; OS, osteosarcoma; sh, short hairpin; NC, negative control.

significantly decreased the protein expression levels of ZEB1 and ZEB2 (Fig. 4E-H). These findings suggest that SPRY4-IT1 and miR-101 may modulate OS tumorigenesis by regulating the expression of ZEB1 and ZEB2.

SPRY4-IT1 sponges miR-101 to regulate the expression of $Z E B 1$ and ZEB2. An increasing number of studies have revealed that IncRNAs may sponge miRNAs and thus disinhibit the target genes of these miRNAs (25). To investigate the possibility of a SPRY4-IT1/miR-101/ZEB axis, the functional interactions between SPRY4-IT1 and miR-101, as well as miR-101 and ZEBs, were explored. RNAInter identified a putative miR-101 binding sequence located in SPRY4-IT1 (Fig. 5A). Compared with the scrambled control, miR-101 mimics significantly reduced SPRY4-IT1-WT-mediated relative luciferase activity, whereas transfection with miR-101 inhibitor increased luciferase activity (Fig. 5B). Mutagenesis of the SPRY4-IT1 fragment (SPRY4-IT1-MUT) completely abolished the effects of the miR-101 mimics and inhibitor. These results suggest that SPRY4-IT1 may directly interact with miR-101 in OS cells.

Furthermore, bioinformatics analysis using starBase identified a binding sequence for miR-101 in the ZEB1 3'UTR (Fig. 5C). In addition, ZEB2 3'UTR was also predicted to contain a 6 bp binding site for miR-101 (Fig. 5E). Dual-luciferase assays showed that the relative luciferase activities were significantly downregulated in the ZEB1-WT and ZEB2-WT groups by miR-101 mimic transfection (Fig. 5D and F). In contrast, the miR-101 inhibitor increased the relative luciferase activity in the ZEB1-WT and ZEB2-WT groups (Fig. 5D and F). Mutagenesis of the seed binding sequences completely eliminated the changes induced by miR-101 mimics and inhibitor (Fig. 5D and F). These findings confirmed that miR-101 could directly target ZEB1 and ZEB2 in OS cells and together these data suggest that SPRY4-IT1 may sponge miR-101 and thus disinhibit the expression of ZEB1/2. The proposed SPRY4-IT1/miR-101/ZEB axis represents a novel axis which may modulate tumorigenesis of OS.

SPRY4-IT1 knockdown inhibits cell growth through upregulation of miR-101 in OS. As MG-63 cells exhibited the highest expression levels of SPRY4-IT1 in the OS cells assessed, subsequent experiments on the SPRY4-IT1/miR-101/ZEBs axis was performed in MG-63 cells. In the present study, the effects of miR-101 inhibitor on cell growth induced by SPRY4-IT1 knockdown wanted to be determined. Therefore, shSPRY4-1T1 transfection alone group is an essential control for this experiment, even though it had already been performed in Fig. 4. Thus, this transfection was repeated in Fig. shSPRY4-IT1 transfection significantly reduced the expression of SPRY4-IT1 and increased miR-101 levels (Fig. 4, and 6A and B). Although the miR-101 inhibitor had no effect on SPRY4-IT1 expression, transfection of shSPRY4-IT1 with the inhibitors significantly reversed the 

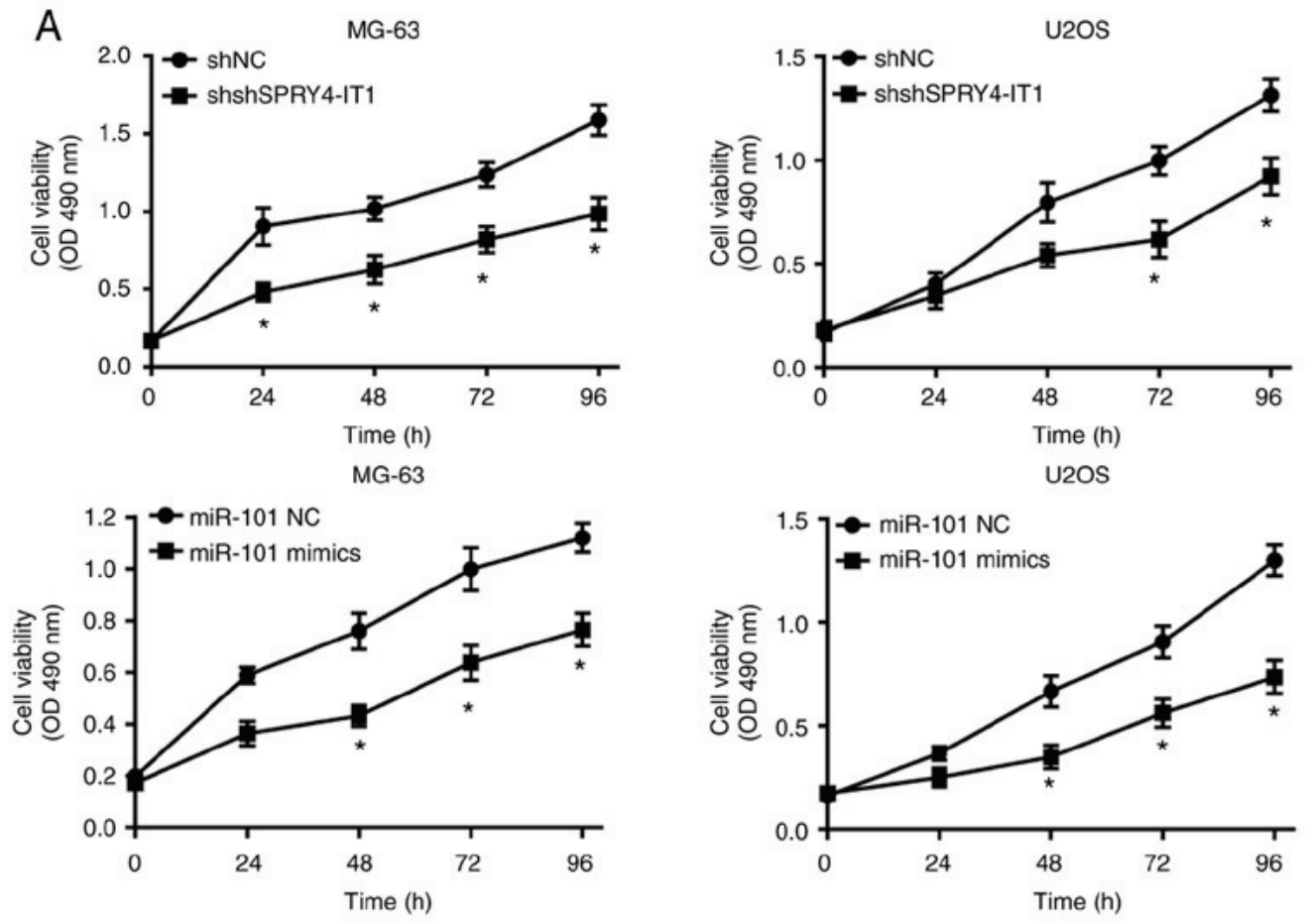

B

MG-63

$\operatorname{shNC}$

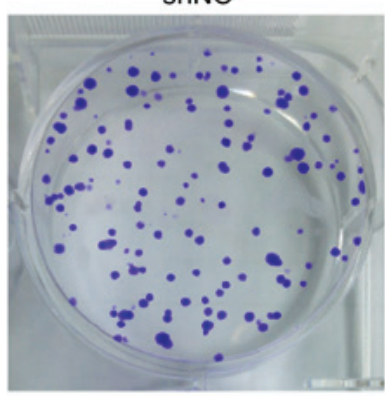

miR-101 NC

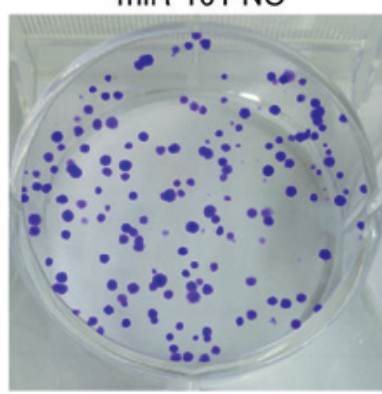

miR-101 mimics

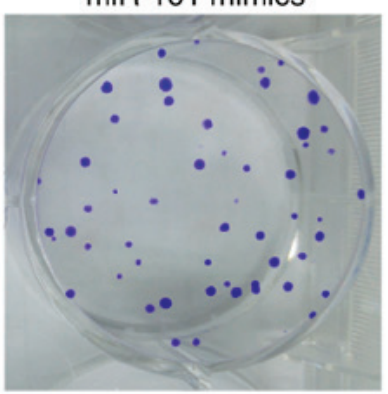

shSPRY4-IT1
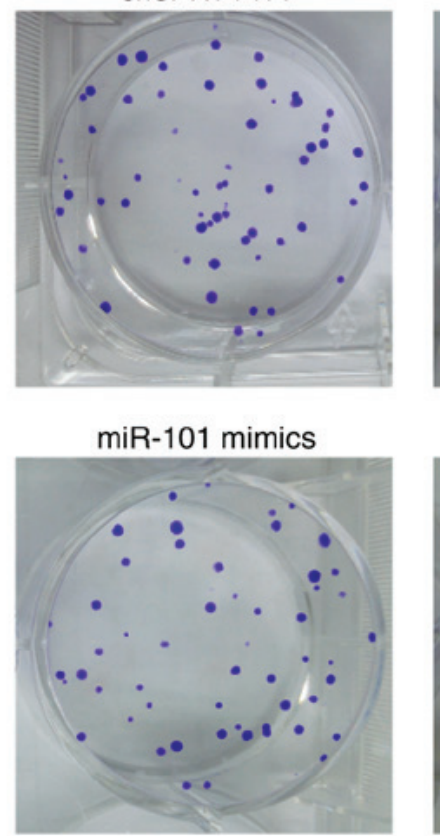

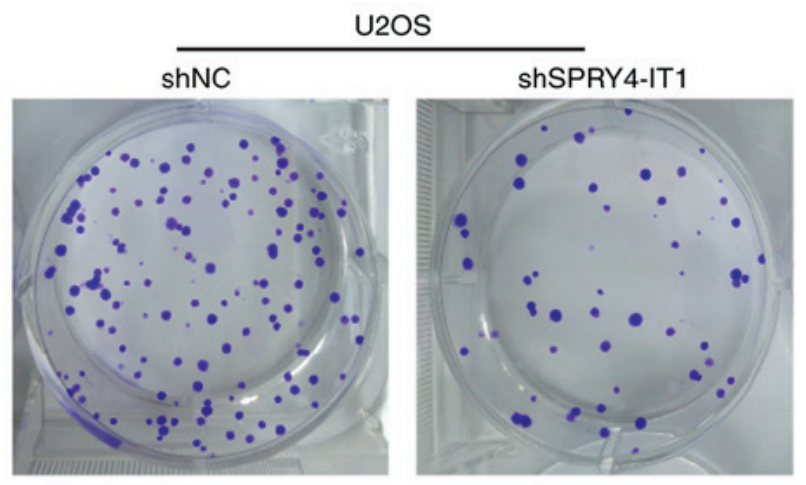

miR-101 NC

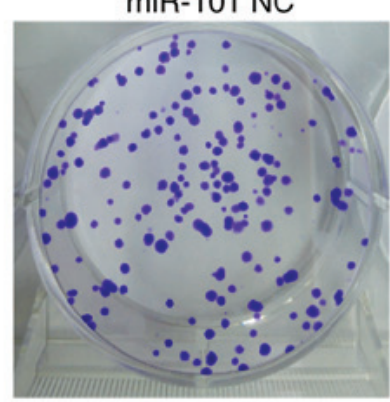

miR-101 mimics

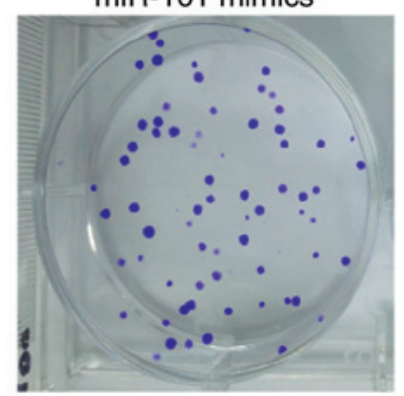

C

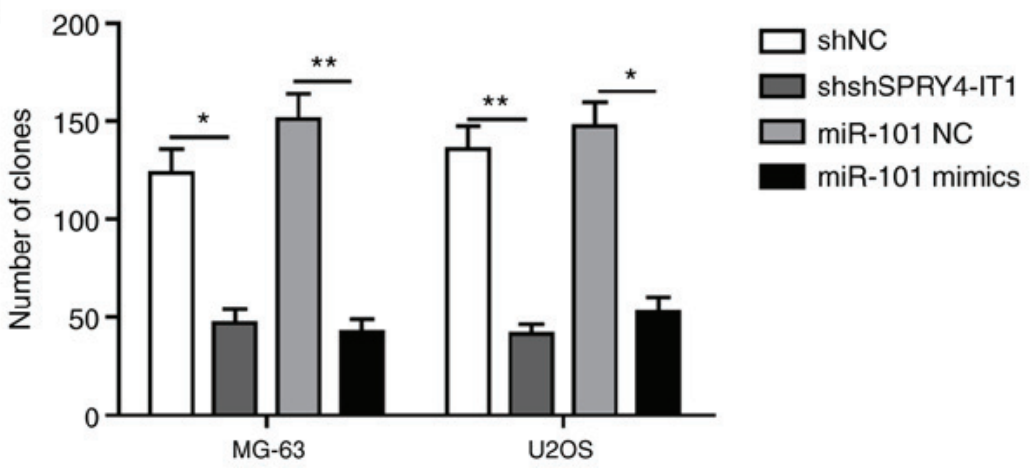

Figure 2. SPRY4-IT1 silencing or miR-101 overexpression reduces OS cell growth. (A) SPRY4-IT1 knockdown or miR-101 overexpression significantly decreased proliferation of MG-63 and U2OS cells as shown using an MTT assay. (B and C) SPRY4-IT1 knockdown or miR-101 overexpression significantly reduced colony formation in MG-63 and U2OS cells. Data are presented as the mean \pm standard deviation of three independent experiments. ${ }^{*} \mathrm{P}<0.05,{ }^{* * *} \mathrm{P}<0.01$. 


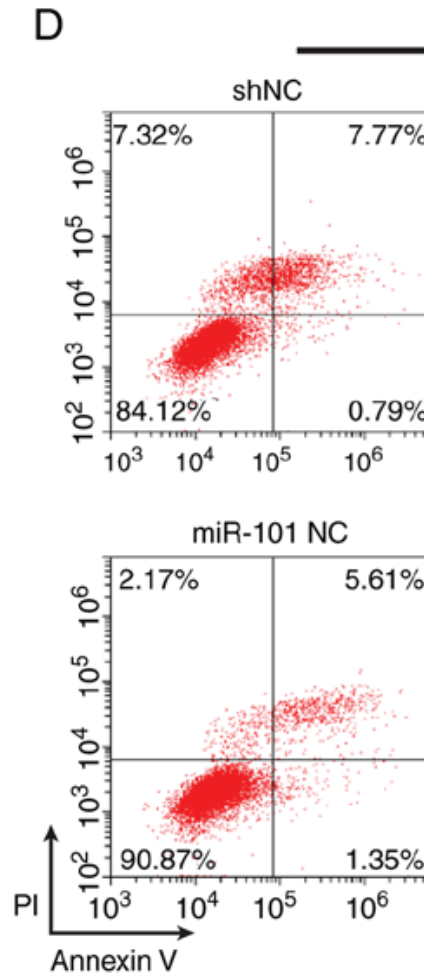

MG-63
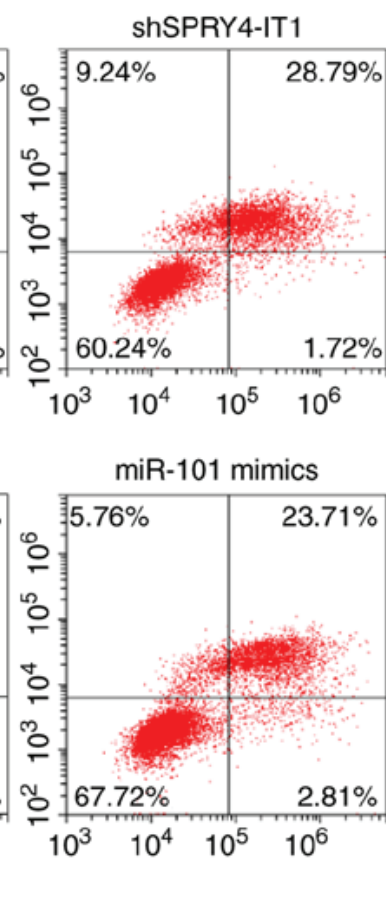
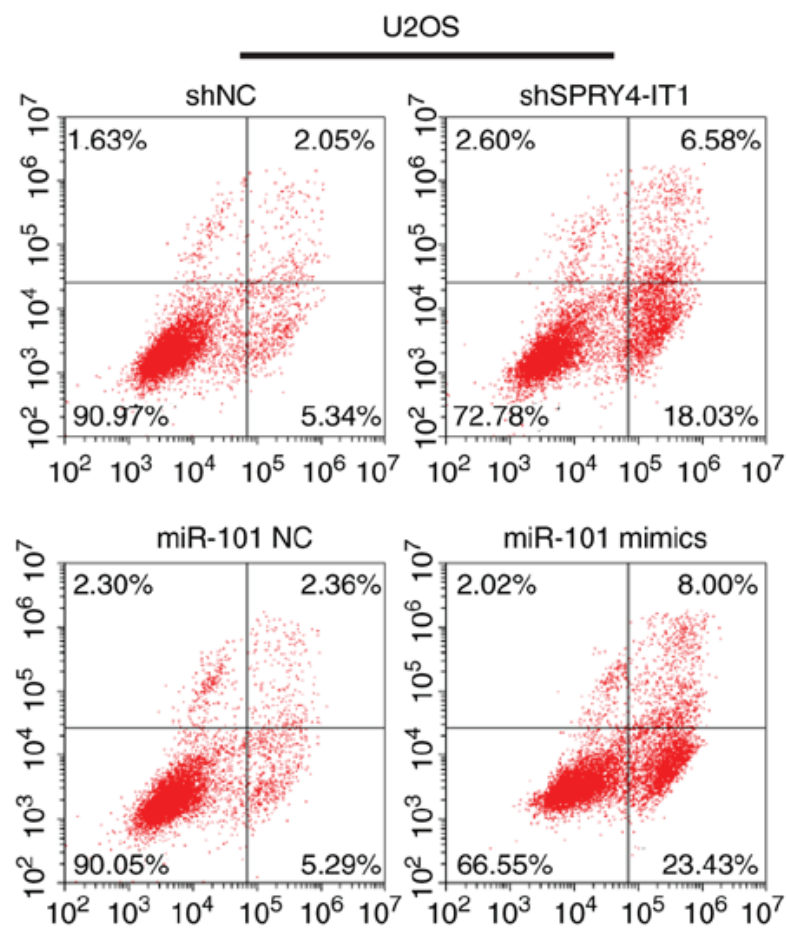

E
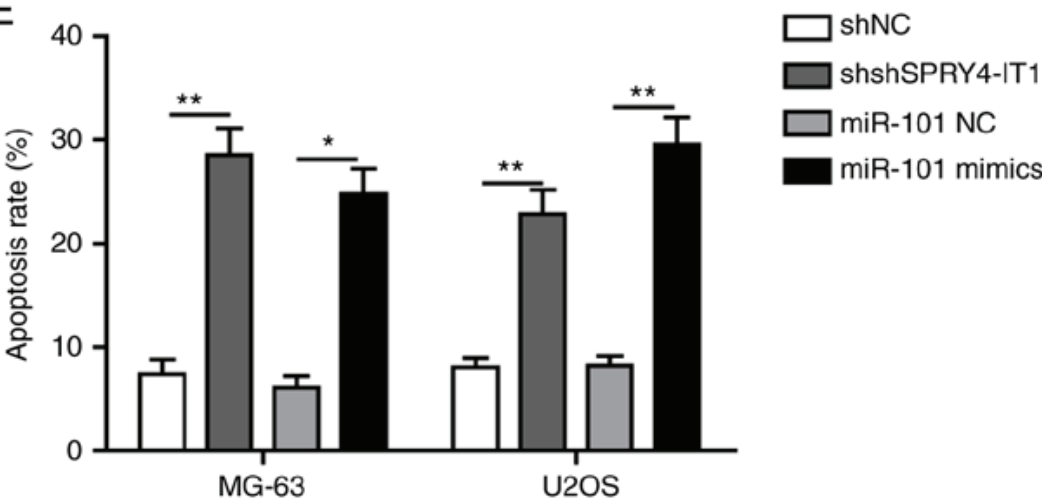

Figure 2. Continued. SPRY4-IT1 silencing or miR-101 overexpression reduces OS cell growth. (D and E) SPRY4-IT1 knockdown or miR-101 overexpression significantly increased apoptosis in both OS cell lines. Data are presented as the mean \pm standard deviation of three independent experiments. ${ }^{\mathrm{P}}<0.05$, ${ }^{* *} \mathrm{P}<0.01$.

miR-101 upregulation induced by SPRY4-IT1 knockdown (Fig. 6A and B). Consistent with Fig. 4E-H, shSPRY4-IT1 reduced the protein levels of both $\mathrm{ZEB} 1$ and $\mathrm{ZEB} 2$ (Fig. 6C-D). However, these effects were partially reversed by co-transfection with miR-101 inhibitors (Fig. 6C and D). Knockdown of SPRY4-IT1 significantly reduced cell growth and co-transfection with miR-101 inhibitor partially reversed the inhibitory effects of shSPRY4-IT1 (Fig. 6E). Similar effects were also observed in the colony formation assay (Fig. 6F and G) where transfection of miR-101 inhibitor reversed the inhibitory effects of shSPRY4-IT1 on the colony formation of OS cells. Finally, an apoptosis assay also revealed that the increase in apoptosis induced by SPRY4-IT1 knockdown was also reversed by co-transfection with the miR-101 inhibitor (Fig. 6H and I). Overall, SPRY4-IT1 knockdown inhibited cell growth and promoted cell apoptosis through upregulation of miR-101. Inhibition of miR-101 in the shSPRY4-IT1 transfected cells abolished the anticancer effects of SPRY4-IT1 knockdown. These results suggested that miR-101 may be a direct downstream effector of SPRY4-IT1.

miR-101 inhibition reverses the shSPRY4-IT1-mediated suppression of cell migration and invasion. As miR-101 inhibition in the shSPRY4-IT1 transfected cells reversed the effects SPRY4-IT1 knockdown on ZEB expression, colony formation and apoptosis, the effects on migration and invasion were determined using the co-transfected cells. Transfection of shSPRY4-IT1 treatment reduced wound closure, whereas inhibition of miR-101 alone promoted wound closure. Co-transfection of the miR-101 inhibitor reversed the inhibitory effects of shSPRY4-IT1 on wound closure (Fig. 7A and B). In the Transwell invasion assay, SPRY4-IT1 knockdown significantly reduced the number of invaded cells and inhibition of miR-101 in the SPRY4-IT1 knockdown cells partially restored the invasive capacity of cells (Fig. 7C and 7D). These data 

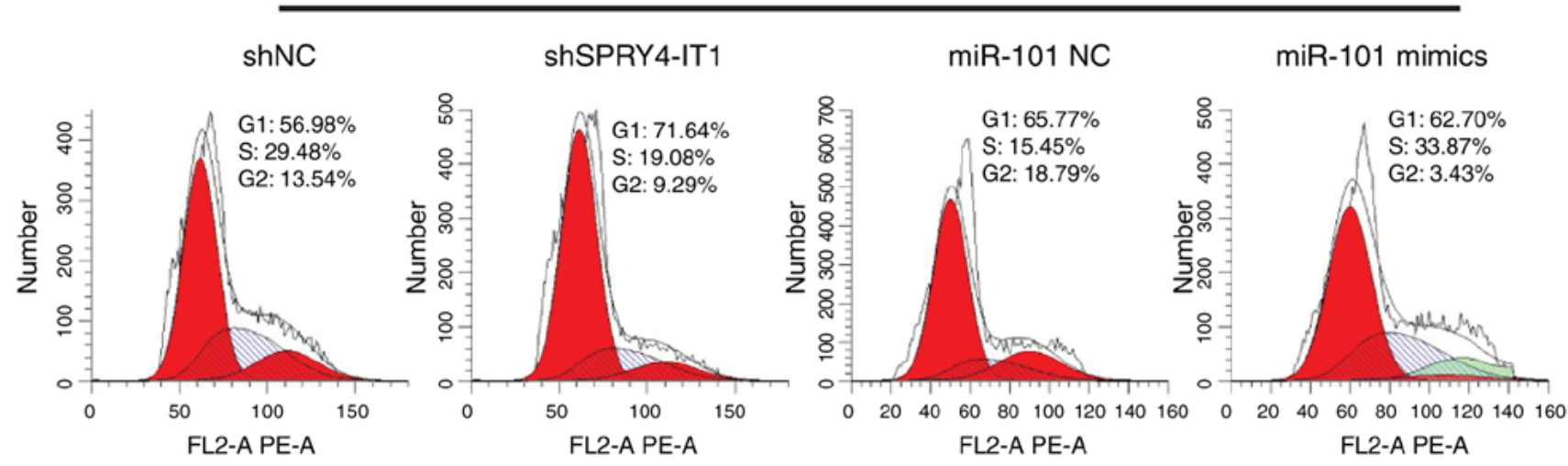

U2OS
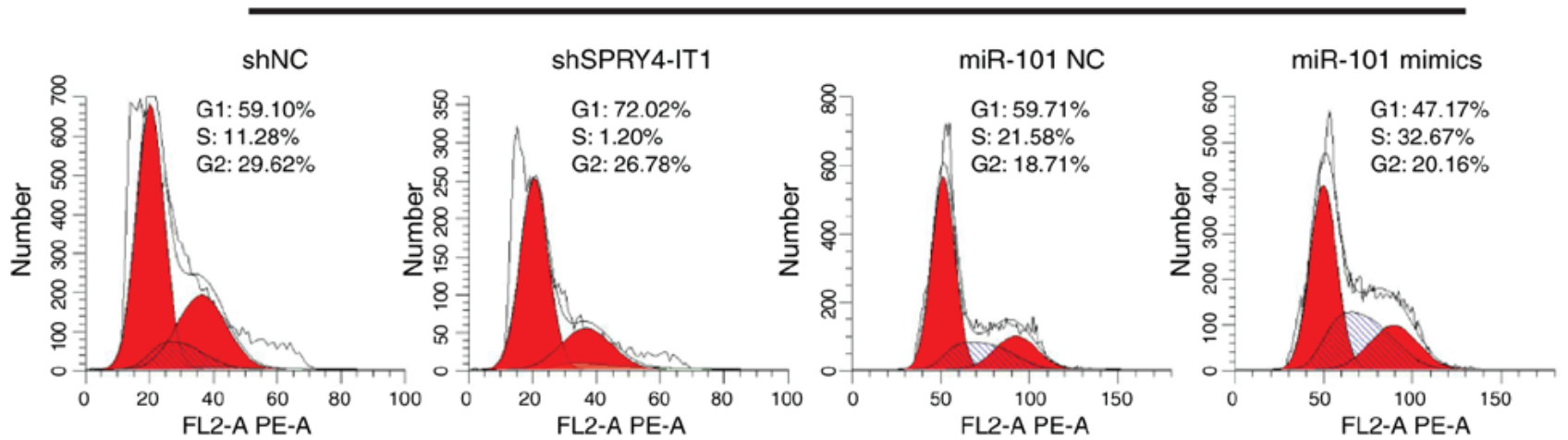

G
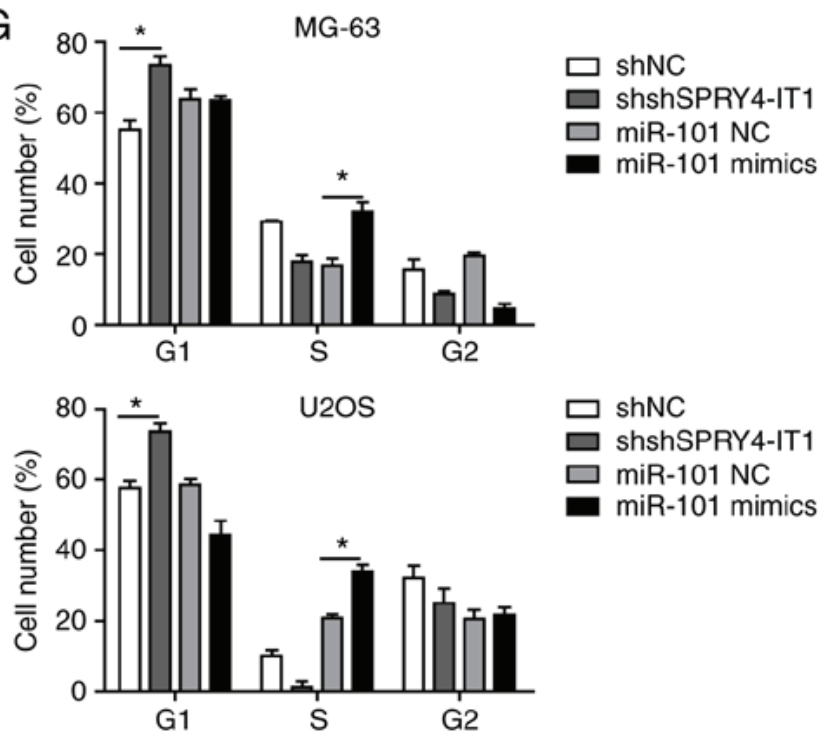

miR-101 mimics

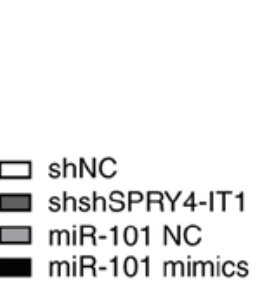
resulted in significant arrest of the cell cycle in MG-63 and U2OS cells. Data are presented as the mean \pm standard deviation of three independent experiments. ${ }^{*} \mathrm{P}<0.05,{ }^{* *} \mathrm{P}<0.01$. SPRY4-IT1, sprouty receptor tyrosine kinase signalling antagonist 4-intronic transcript 1; miR, microRNA; OS, osteosarcoma; sh, short hairpin; NC, negative control; OD, optical density; PI, propidium iodide.

suggest that SPRY4-IT1 may alter the migratory and invasive capacities of OS cells by regulating miR-101 expression.

EMT serves a critical role in cell migration and invasion $(26,27)$, and E-cadherin has been reported to reduce cell migration in a number of different types of cancer (26). ZEB1/2 increase migration and invasion by transcriptionally repressing E-cadherin expression (28). As shown in Fig. 7E and F, SPRY4-IT1 knockdown significantly increased the protein expression levels of E-cadherin. In contrast, transfection of miR-101 inhibitor alone was sufficient to decrease E-cadherin protein expression levels. Upregulation of E-cadherin by shSPRY4-IT1 was partially reversed by co-transfection with the miR-101 inhibitor. The expression levels of vimentin, fibronectin, N-cadherin, MMP-2 and MMP-9, proteins which may promote cell migration and invasion, were also detected. SPRY4-IT1 knockdown reduced the expression of all these 
A
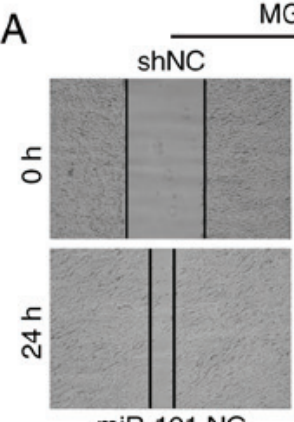

miR-101 NC
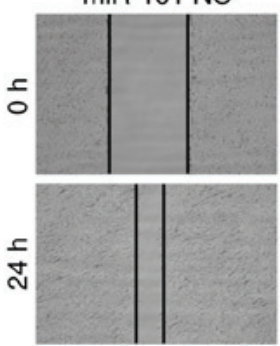

MG-63
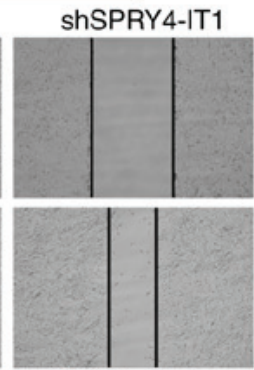

miR-101 mimics

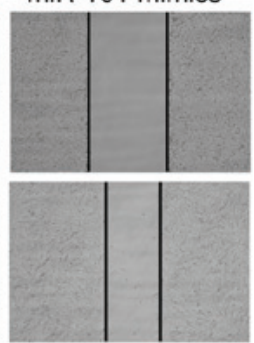

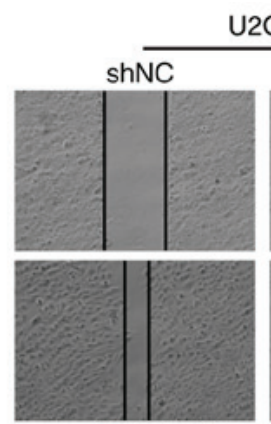

miR-101 NC
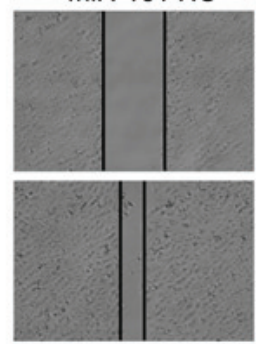

2OS
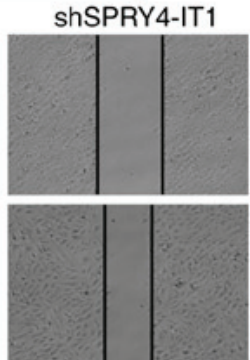

miR-101 mimics
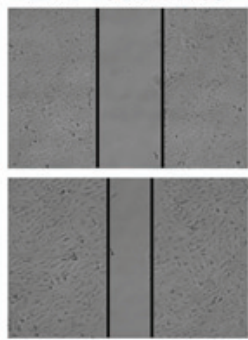

B $\square \operatorname{shNC}$
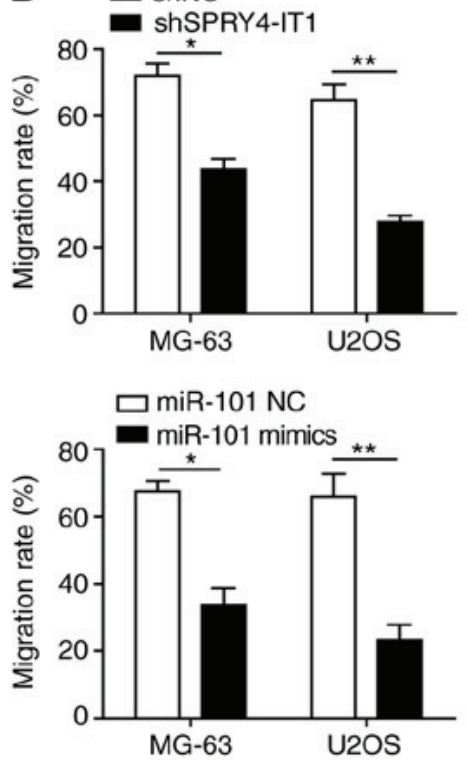

C

MG-63
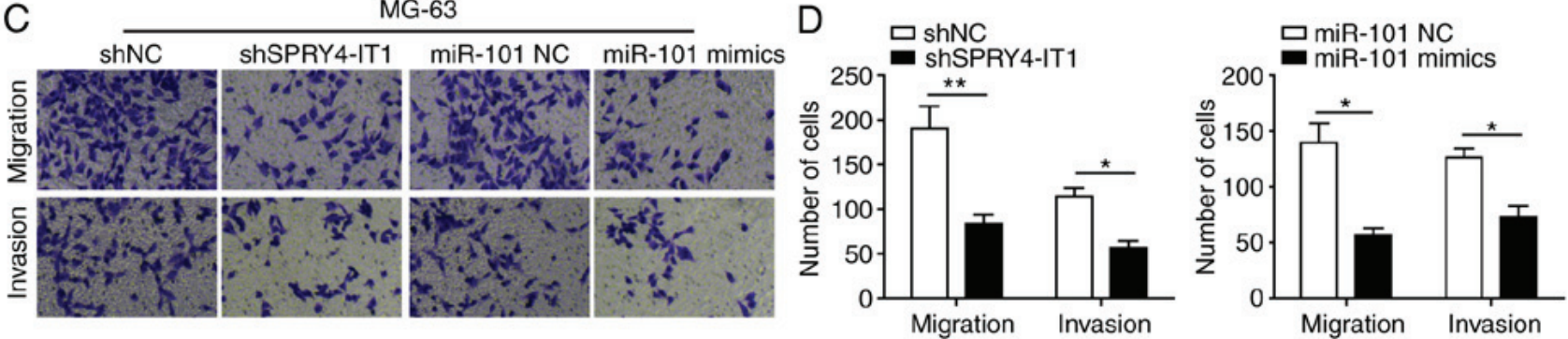

E
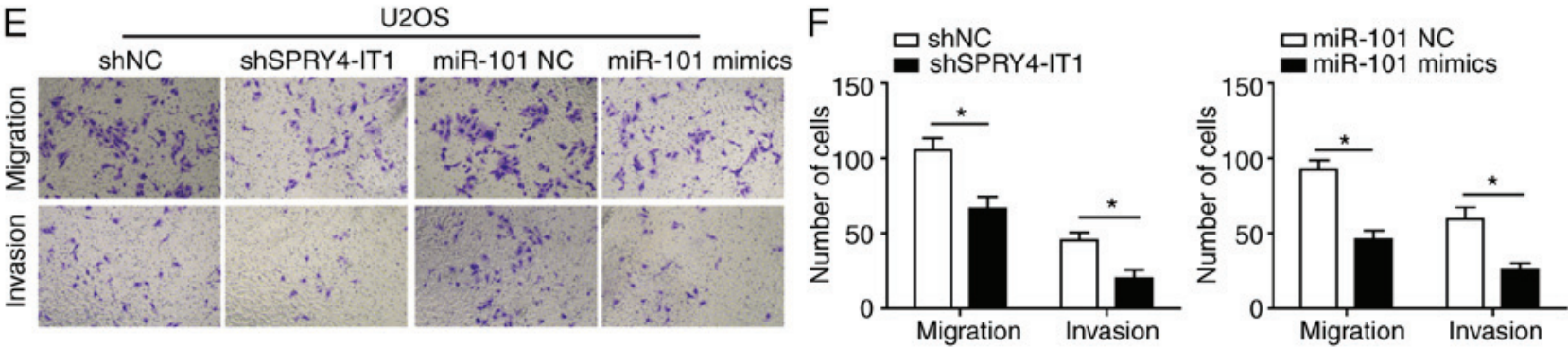

Figure 3. SPRY4-IT1 knockdown or miR-101 overexpression reduces the migratory and invasive capacity of OS cells. (A and B) SPRY4-IT1 knockdown or miR-101 overexpression significantly reduced the migratory rate of MG-63 and U2OS cells in the wound healing assay. SPRY4-IT1 knockdown or miR-101 overexpression reduced Transwell migration and invasion of (C and D) MG-63 and (E and F) U2OS cells. Data are presented as the mean \pm standard deviation of three independent experiments. ${ }^{*} \mathrm{P}<0.05,{ }^{* *} \mathrm{P}<0.01$. SPRY4-IT1, sprouty receptor tyrosine kinase signalling antagonist 4 -intronic transcript 1 ; miR, microRNA; OS, osteosarcoma; sh, short hairpin; NC, negative control.

proteins, whereas miR-101 inhibition increased their expression. Inhibition of miR-101 in the shSPRY4-IT1 cells was sufficient to reverse the changes in the expression of all these proteins. Based on these results, it was hypothesized that SPRY4-IT1 promoted cell migration and invasion by sponging miR-101, which subsequently disinhibited ZEB1/2, leading to the upregulation of the EMT-associated proteins.

SPRY4-IT1 knockdown decreases tumour growth in vivo. The anti-tumour function of SPRY4-IT1 knockdown was evaluated in a xenograft tumour model. MG-63 and U2OS cells stably transfected with shSPRY4-IT1 or shNC were subcutaneously injected into nude mice. A total of 30 days after model establishment, the mice were sacrificed for tumour collection
(Fig. 8A). Knockdown of SPRY4-IT1 was sufficient to reduce tumour growth of both MG-63 and U2OS OS cells in vivo, as indicated by the significant reduction in tumour volume and weight (Fig. 8B and C). Tumours were lysed for qPCR and western blot analysis. SPRY4-IT1 expression levels were significantly lower in the tumours of mice injected with the shSPRY4-IT1 cells (Fig. 8D), and miR-101 expression was increased in the tumours (Fig. 8E). ZEB1 and ZEB2 mRNA (Fig. 8F and G) and protein expression levels (Fig. 8H and I) were significantly reduced in the tumours of mice injected with the shSPRY4-IT1 cells, and the expression of E-cadherin, a target gene of ZEB1 and ZEB2, was significantly increased (Fig. 8H and I). These results demonstrate that a dysregulated SPRY4-IT1/miR-101/ZEBs axis may promote tumour growth 
A

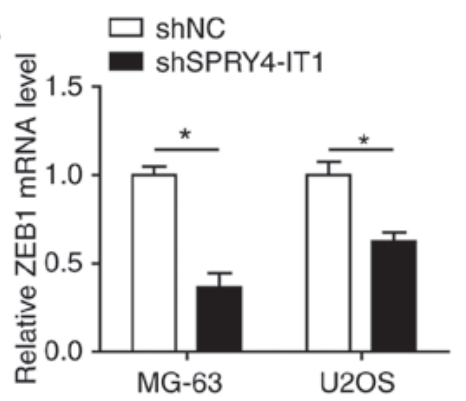

B

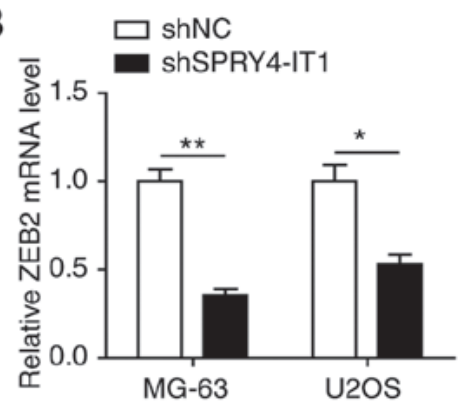

G

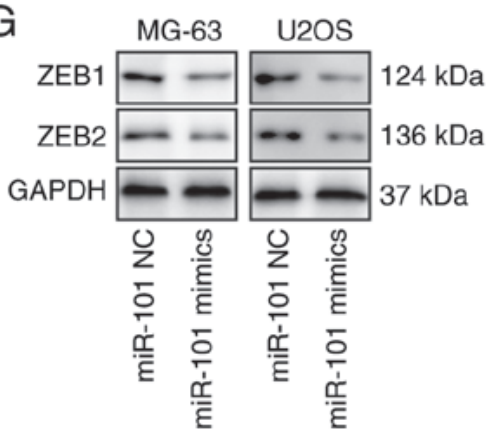

C

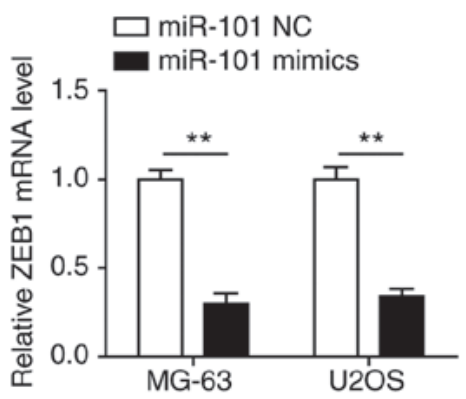

D

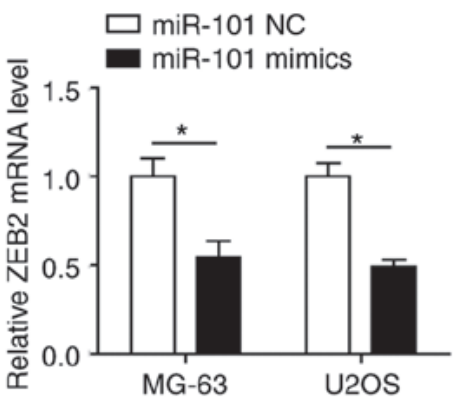

E

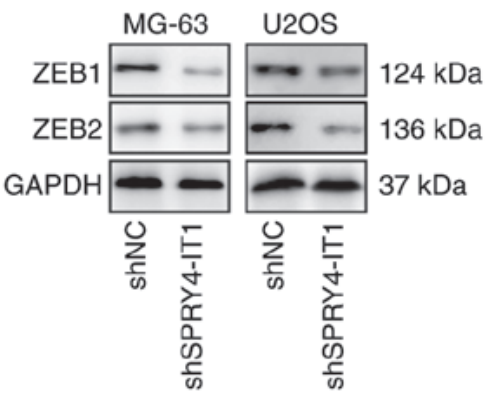

F

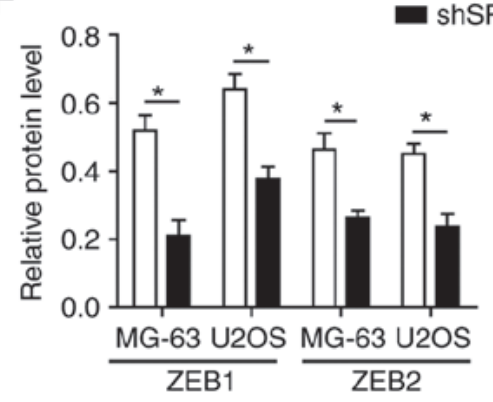

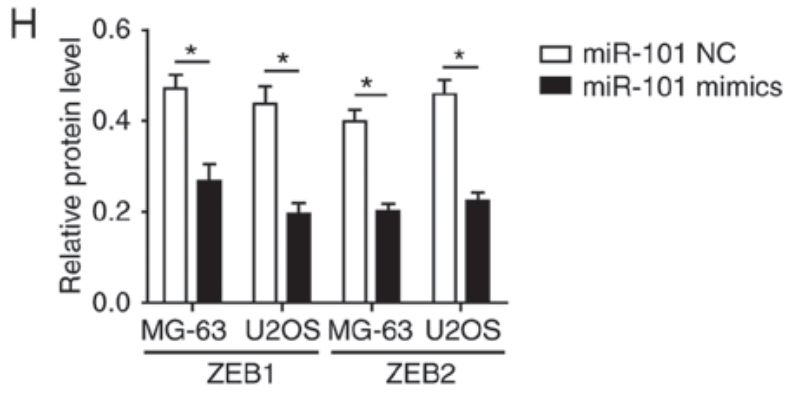

Figure 4. SPRY4-IT1 and miR-101 modulate the expression of ZEB1 and ZEB2 in OS cells. shSPRY4-IT1 significantly decreased the mRNA expression levels of (A) ZEB1 and (B) ZEB2 in MG-63 and U2OS cells. miR-101 overexpression significantly decreased the mRNA expression levels of (C) ZEB1 and (D) ZEB2 in MG-63 and U2OS cells. (E and F) Western blotting demonstrated that SPRY4-IT1 knockdown decreased ZEB1 and ZEB2 protein expression levels in MG-63 and U2OS cells. (G and H) Western blotting demonstrated that miR-101 overexpression decreased ZEB1 and ZEB2 protein expression levels in MG-63 and U2OS cells. Data are presented as the mean \pm standard deviation of three independent experiments. ${ }^{*} \mathrm{P}<0.05,{ }^{* *} \mathrm{P}<0.01$. SPRY4-IT1, sprouty receptor tyrosine kinase signalling antagonist 4-intronic transcript 1; miR, microRNA; OS, osteosarcoma; sh, short hairpin; NC, negative control; ZEB, zinc finger E-box-binding homeoboxes.

in vivo and targeting this axis highlights a potentially novel approach for treating patients with OS.

\section{Discussion}

OS, the most frequent type of malignant bone tumour, often afflicts children and accounts for $2.4 \%$ of all malignancies and $20 \%$ of all types of primary bone cancer in paediatric patients $(1,2)$. Despite considerable advances in treatment and diagnosis, the survival rates of patients with OS remains poor, and the rate of recurrence is as high as $30-50 \%$ for patients with initial localized disease (29). In the present study, SPRY4-IT1 was determined to function as a ceRNA which regulated the expression of the oncogenes ZEB1 and ZEB2 by competing for miR-101 binding in OS cells. Inhibition of SPRY4-IT1 restored the expression of miR-101, which then inhibited cell growth, migration and invasion by regulating the levels of ZEB1 and ZEB2 in OS cells.
Previous studies have highlighted the diagnostic and/or prognostic values of IncRNAs in various types of cancer $(30,31)$. Non-coding RNAs, including lncRNAs and miRNAs, have been frequently reported to be differentially expressed in the cancer tissues of patients $(30,31)$. Recently, it was reported that the serum levels of miR-101 were significantly lower in patients with OS compared with the healthy controls (32). Therefore, miR-101 may represent a potentially useful biomarker for predicting the survival of patients with OS. Consistent with the previous study, miR-101 expression was demonstrated to be significantly downregulated in three OS cell lines compared with the normal osteoblast cell line in the present study. Expression of SPRY4-IT1, which was recently demonstrated to modulate miR-101 in cholangiocarcinoma (33), was upregulated in OS cells compared with the normal cells. These results suggest that a functional interaction between SPRY4-IT1 and miR-101 may be observed during the pathogenesis of OS. Indeed, knockdown of SPRY4-IT1 was 
A

$\begin{array}{ccc}\text { hsa-miR-101 } & 3^{\prime} \text { AAGUCAAUAGUGUCAUGACAU 5' } \\ \text { SPRY4-IT1-WT } & \text { 5' } & \text { AUCAUUUA - - GCUGUGCUGAG } \\ \text { SPRY4-IT1-MUT } & \text { 5' } & \text { AUCAUUUA - - GCUCAUGACAG }\end{array}$

B
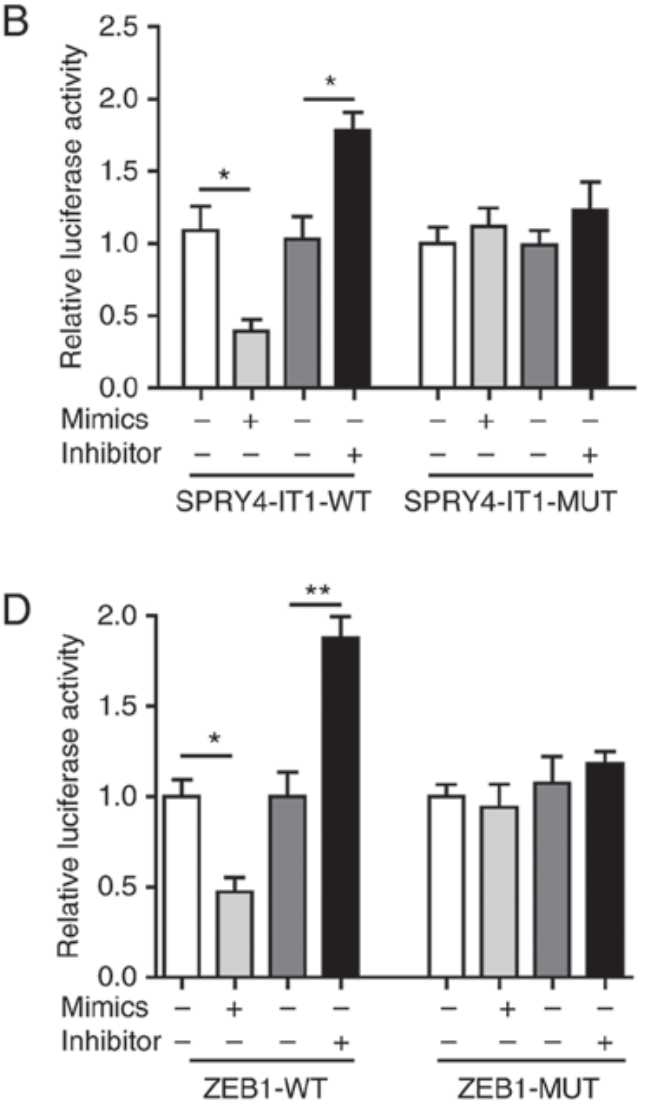

$\mathrm{F}$

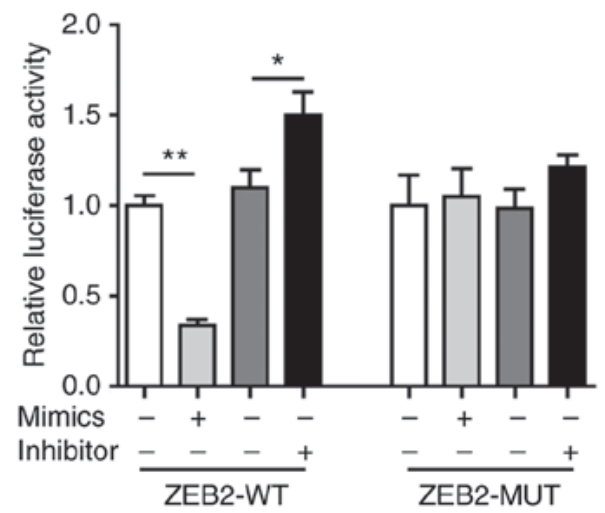

Figure 5. SPRY4-IT1 sponges miR-101 to regulate the expression of ZEB1 and ZEB2. (A) Predicted binding site between SPRY4-IT1 and miR-101.(B) miR-101 mimics reduced, whereas miR-101 inhibitor increased, luciferase activity in the SPRY4-IT1-WT group. Luciferase activity was not altered by miR-101 mimics or inhibitors in the SPRY4-IT1-MUT group. (C) Predicted binding site between miR-101 and ZEB1. (D) miR-101 mimics reduced, whereas miR-101 inhibitor increased, luciferase activity in the ZEB1-WT group. Luciferase activity was not altered by miR-101 mimics or inhibitors in the ZEB1-MUT group. (E) Predicted binding site between miR-101 and ZEB2. (F) miR-101 mimics reduced, whereas miR-101 inhibitor increased, luciferase activity in the ZEB2-WT group. Luciferase activity was not altered by miR-101 mimics or inhibitors in the ZEB2-MUT group. Data are presented as the mean \pm standard deviation of three independent experiments. $\mathrm{P}<0.05,{ }^{* *} \mathrm{P}<0.01$. SPRY4-IT1, sprouty receptor tyrosine kinase signalling antagonist 4-intronic transcript 1; miR, microRNA; ZEB, zinc finger E-box-binding homeoboxes.

sufficient to restore miR-101 levels in OS cells. However, overexpression of miR-101 did not affect SPRY4-IT1 levels, implying that SPRY4-IT1 was the upstream modulator of miR-101. Furthermore, in silico analysis indicated that SPRY4-IT1 may bind to a complementary sequence in miR-101. This binding was confirmed using a dual-luciferase assay, which showed that the relative luciferase activity of the SPRY4-IT1-WT group was reduced in the presence of miR-101 mimics. Therefore, it was plausible that SPRY4-IT1 sponged miR-101, resulting in the disruption of miR-101-mediated tumour suppression in OS.
To test the hypothesis that SPRY4-IT1 acted as a sponge of miR-101, the effect of SPRY4-IT1 and miR-101 interactions on cancer cells was further investigated. shSPRY4-IT1 or miR-101 mimic transfection was used to study the functional effects of SPRY4-IT1 and miR-101, respectively. Knockdown of SPRY4-IT1 alone was sufficient to decrease cell growth, cause cell cycle arrest and induce apoptosis in OS cells. Wound healing and Transwell assays also showed that shSPRY4-IT1 attenuated cell migration and invasion. This was further confirmed by the upregulation of the epithelial marker 


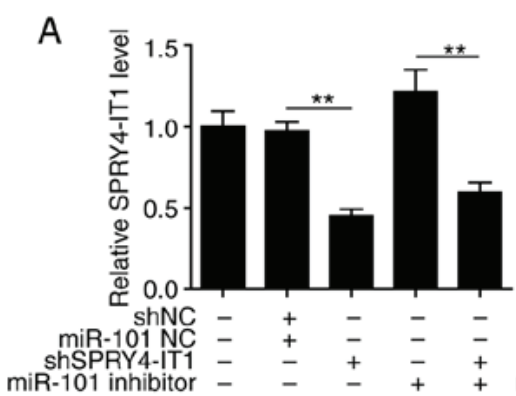

B

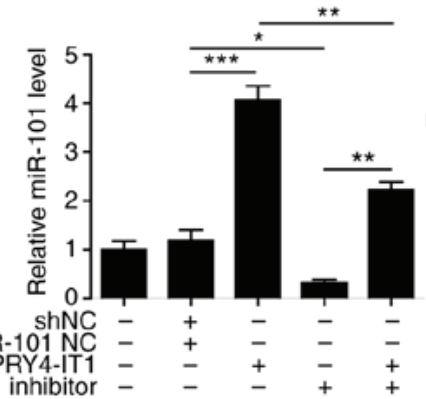

D

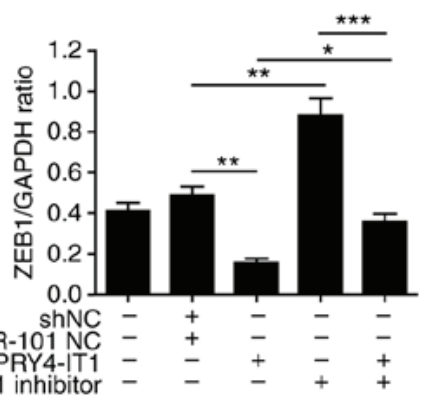

$\mathrm{F}$

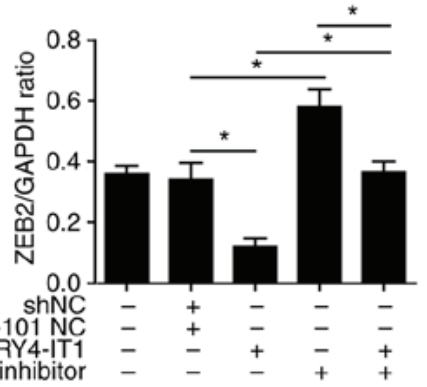

ShSPRY4-1T

miR-101 inhibitor

shSPRY4-IT1+

E

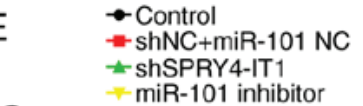

E $2.5 \multimap \rightarrow$ shSPRY4-IT1+miR-101 inhibitor
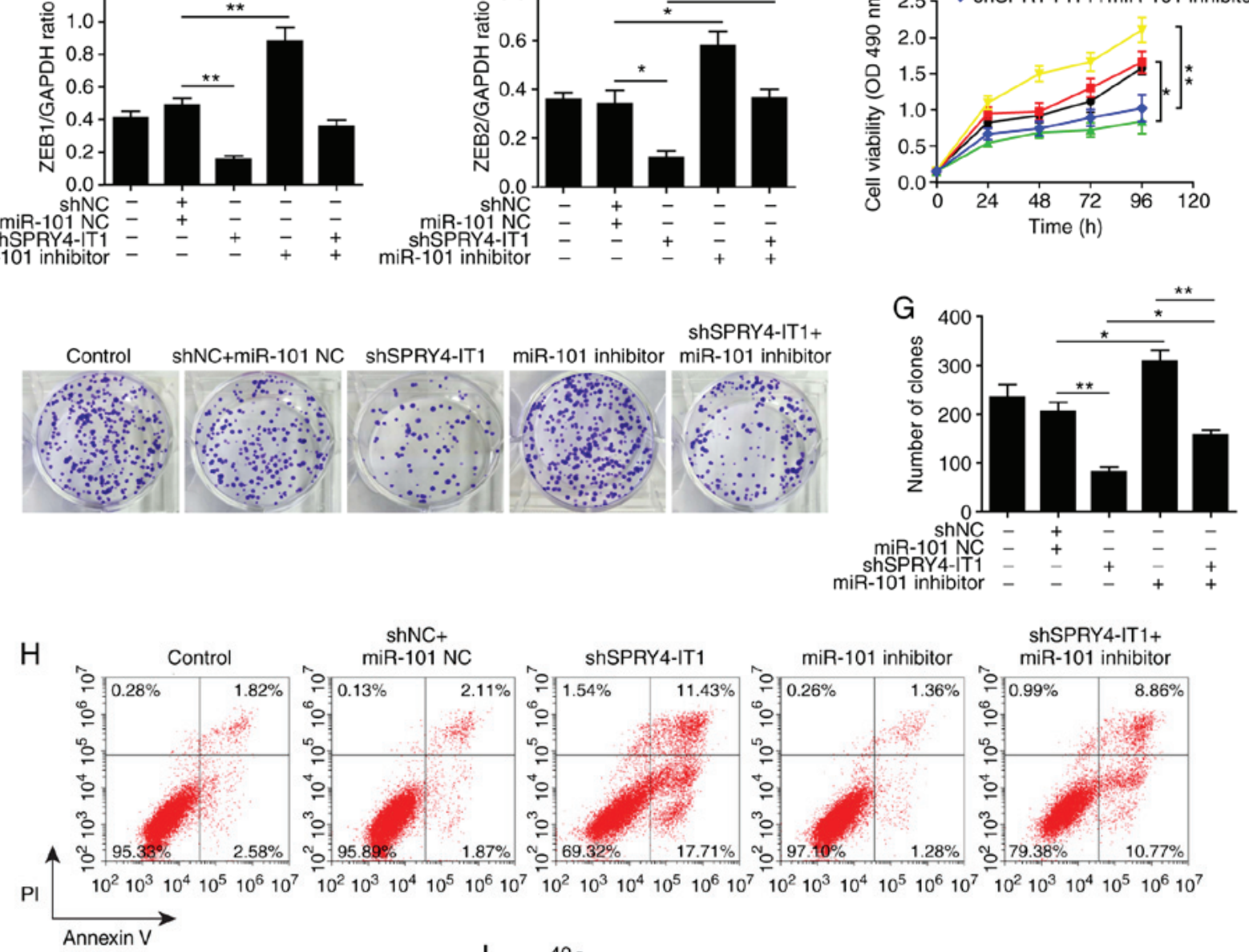

ShSPRY4-IT1+

miR-101 inhibitor

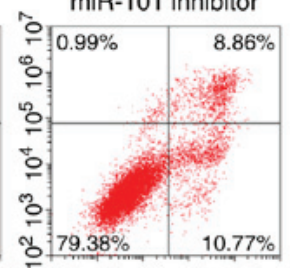

$10^{2} 10^{3} 10^{4} 10^{5} 10^{6} 10^{7} 10^{2} 10^{3} 10^{4} 10^{5} 10^{6} 10^{7} 10^{2} 10^{3} 10^{4} 10^{5} 10^{6} 10^{7} 10^{2} 10^{3} 10^{4} 10^{5} 10^{6} 10^{7}$

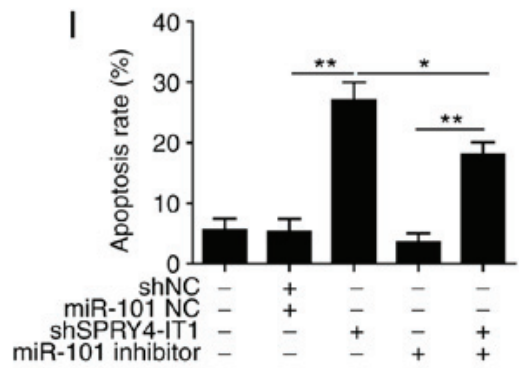

Figure 6. SPRY4-IT1 knockdown reduces cell growth through upregulation of miR-101 in MG-63 cells. (A) Transfection of shSPRY4-IT1 significantly decreased SPRY4-IT1 mRNA expression levels, whereas transfection of miR-101 inhibitor did not affect SPRY4-IT1 mRNA expression levels. (B) SPRY4-IT1 knockdown significantly increased, whereas miR-101 inhibitor significantly reduced, miR-101 levels. The increase in miR-101 induced by shSPRY4-IT1 was significantly reversed by simultaneous transfection of miR-101 inhibitors. (C and D) SPRY4-IT1 knockdown significantly decreased, whereas miR-101 inhibitor increased ZEB1 and ZEB2 protein expression levels. The reduction in ZEB1 and ZEB2 by shSPRY4-IT1 was partially abolished by transfection of miR-101 inhibitor. (E) SPRY4-IT1 knockdown decreased OS cell growth in the MTT assay, and simultaneous transfection with miR-101 inhibitor reversed the effects of shSPRY4-IT1 on cell growth. (F and G) SPRY4-IT1 knockdown significantly decreased, whereas miR-101 inhibitor increased colony formation. Reduced colony formation in cells transfected with shSPRY4-IT1 was partially abolished by simultaneous transfection with miR-101 inhibitor. (H and I) SPRY4-IT1 knockdown significantly increased, whereas miR-101 inhibitor decreased apoptosis. shSPRY4-IT1-induced apoptosis was partially reversed by simultaneous transfection with miR-101 inhibitor. Data are presented as the mean \pm standard deviation of three independent experiments. "P $<0.05$, ${ }^{* *} \mathrm{P}<0.01$. SPRY4-IT1, sprouty receptor tyrosine kinase signalling antagonist 4-intronic transcript 1; miR, microRNA; OS, osteosarcoma; sh, short hairpin; NC, negative control; OD, optical density; PI, propidium iodide; ZEB, zinc finger E-box-binding homeoboxes. 


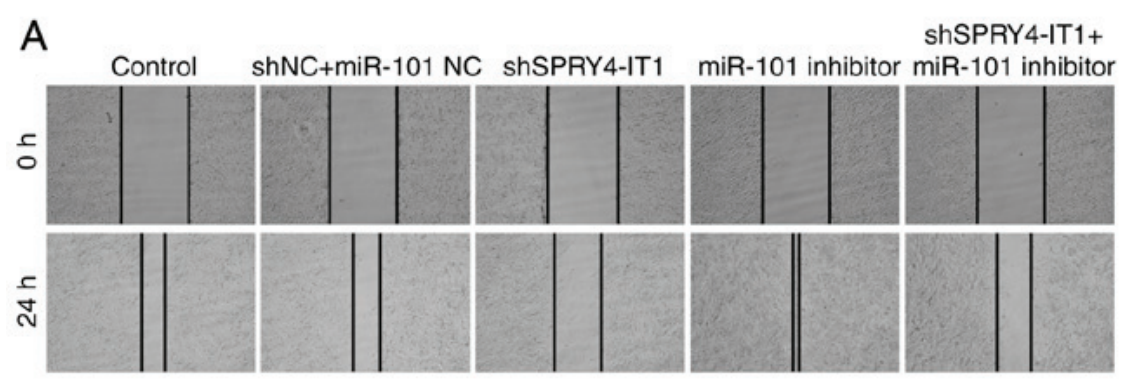

B
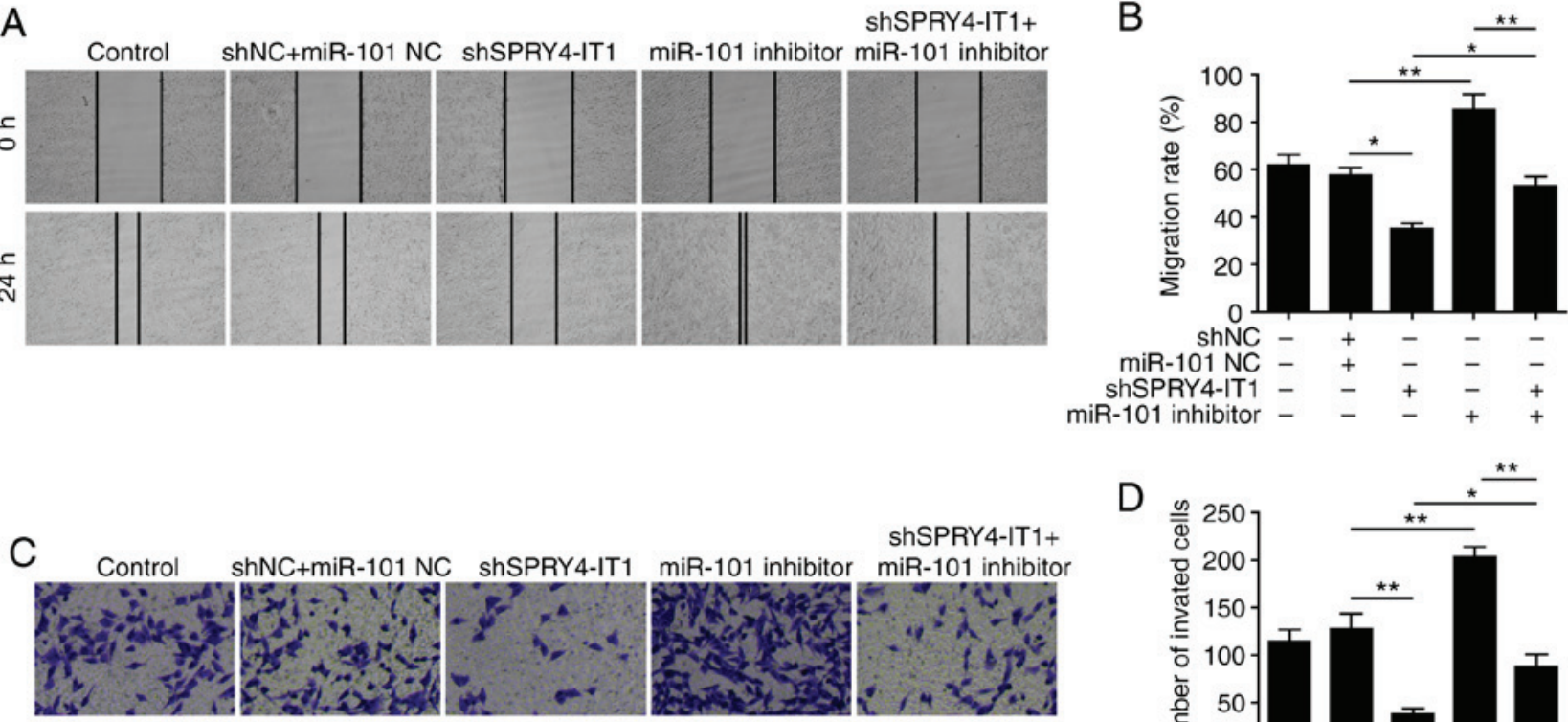

ShSPRY4-IT1+

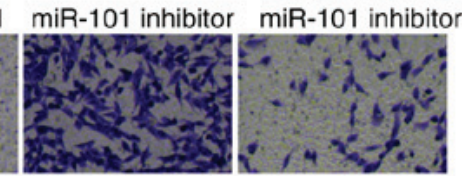

D

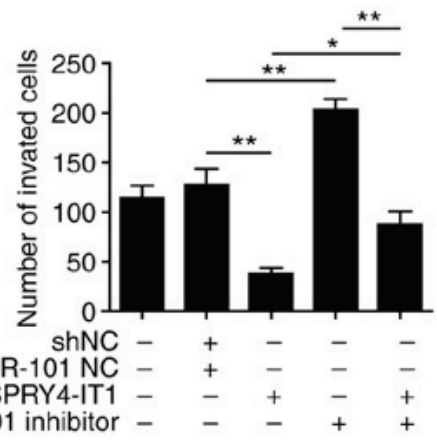

E

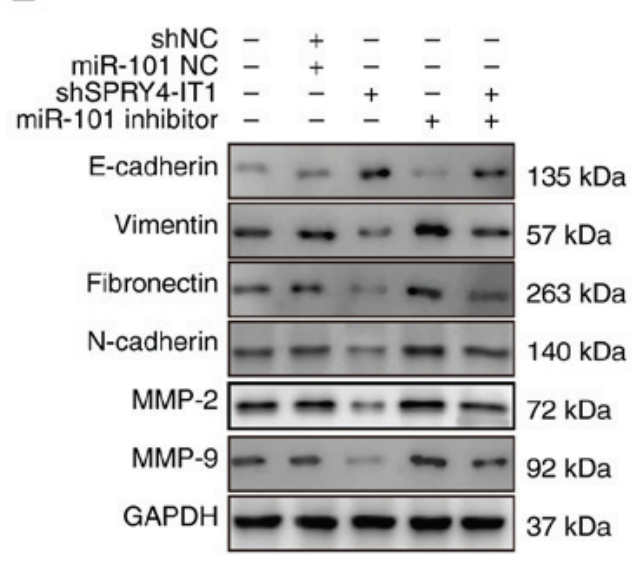

$\mathrm{F}$
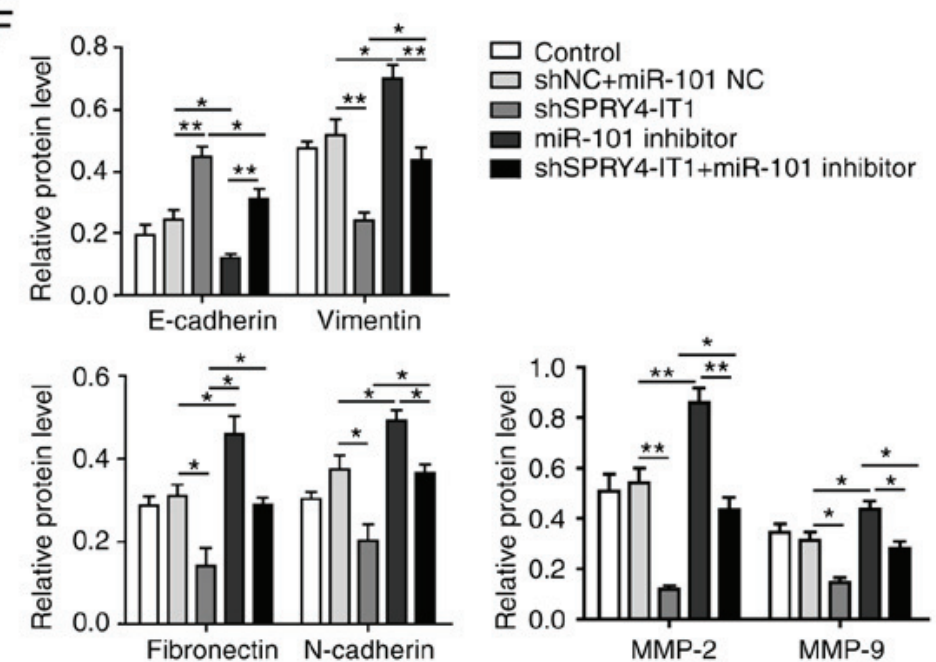

Figure 7. miR-101 inhibitor reverses the shSPRY4-IT1-mediated suppression of cell migration and invasion in MG-63 cells. (A and B) SPRY4-IT1 knockdown significantly delayed wound closure, whereas miR-101 inhibitor increased wound closure. The shSPRY4-IT1-induced decrease in migratory rate was partially reversed by miR-101 inhibitor. (C and D) SPRY4-IT1 knockdown significantly reduced cell invasion, whereas miR-101 inhibitor increased cell invasion. miR-101 expression in SPRY4-IT1 knockdown cells reversed the effects of SPRY4-IT1 knockdown on cell invasion. (E and F) shSPRY4-IT1 increased E-cadherin protein expression levels, whereas vimentin, fibronectin, N-cadherin, MMP-2 and MMP-9 levels were decreased by SPRY4-IT1 knockdown, whereas cells transfected with miR-101 inhibitors exhibited the opposite changes in protein expression levels of epithelial-mesenchymal transition-associated proteins. Transfection of miR-101 inhibitor reversed the effects of shSPRY4-IT1 transfection. Data are presented as the mean \pm standard deviation of three independent experiments. ${ }^{*} \mathrm{P}<0.05,{ }^{* *} \mathrm{P}<0.01$. SPRY4-IT1, sprouty receptor tyrosine kinase signalling antagonist 4-intronic transcript 1; miR, microRNA; sh, short hairpin; NC, negative control; MMP, matrix metalloproteinase.

E-cadherin and downregulation of the mesenchymal markers vimentin, fibronectin, N-cadherin, MMP-9 and MMP-2 when SPRY4-IT1 was knocked down. Notably, similar anticancer effects were also observed in cells treated with miR-101 mimics, whereas transfection of the miR-101 inhibitor resulted in the opposite outcomes. Through MTT, colony formation, flow cytometry, wound healing and Transwell invasion assays, the effects on cell growth, migration, invasion and cell cycle progression induced by SPRY4-IT1 knockdown were partially abolished when miR-101 was simultaneously inhibited in vitro. These results demonstrate that SPRY4-IT1 sequestered miR-101 in OS and that inhibition of SPRY4-IT1 was sufficient to impede growth of OS through restoration of miR-101 function. The results of the present study are in agreement with previous studies which showed that increased SPRY4-IT1 levels could sponge miR-101 and thus confer oncogenic properties, such as proliferation and invasion, on colorectal cancer and cholangiocarcinoma cells $(33,34)$.

Accompanying these findings, both ZEB1 and ZEB2 mRNA and protein expression levels were reduced by SPRY4-IT1 knockdown or miR-101 overexpression. The effects of shSPRY4-IT1 on ZEB expression were also partially attenuated 

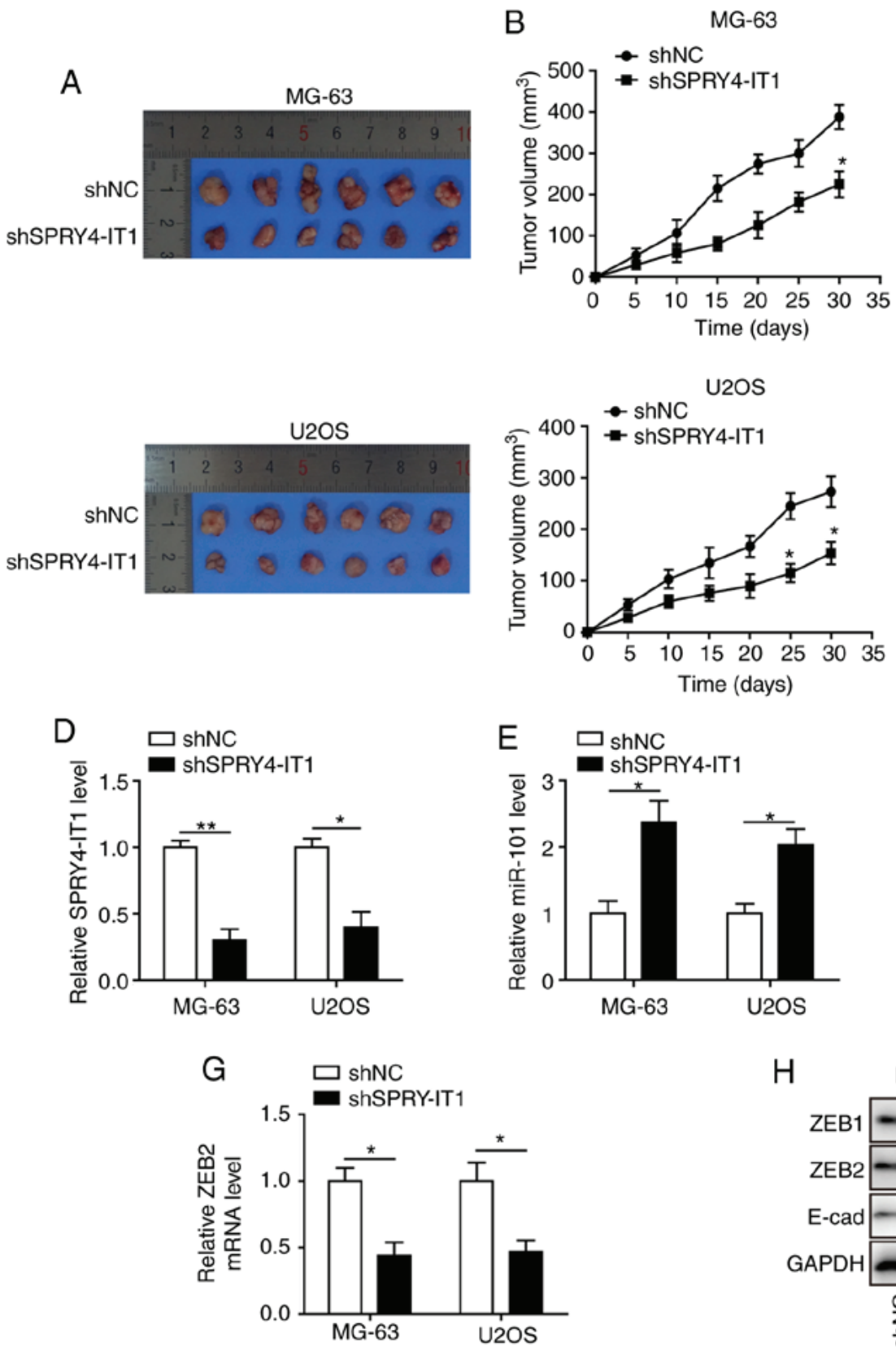

C
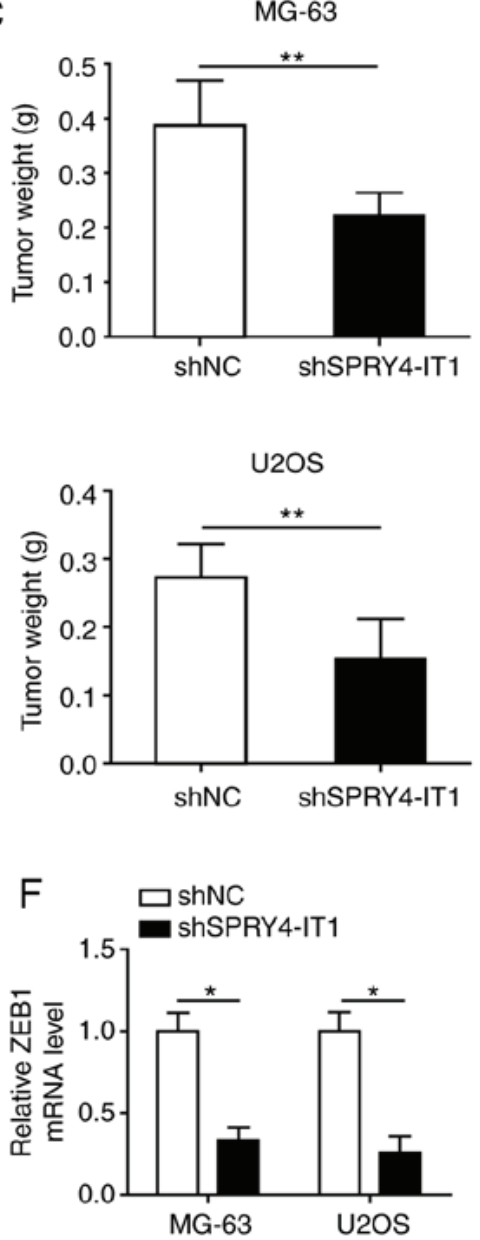
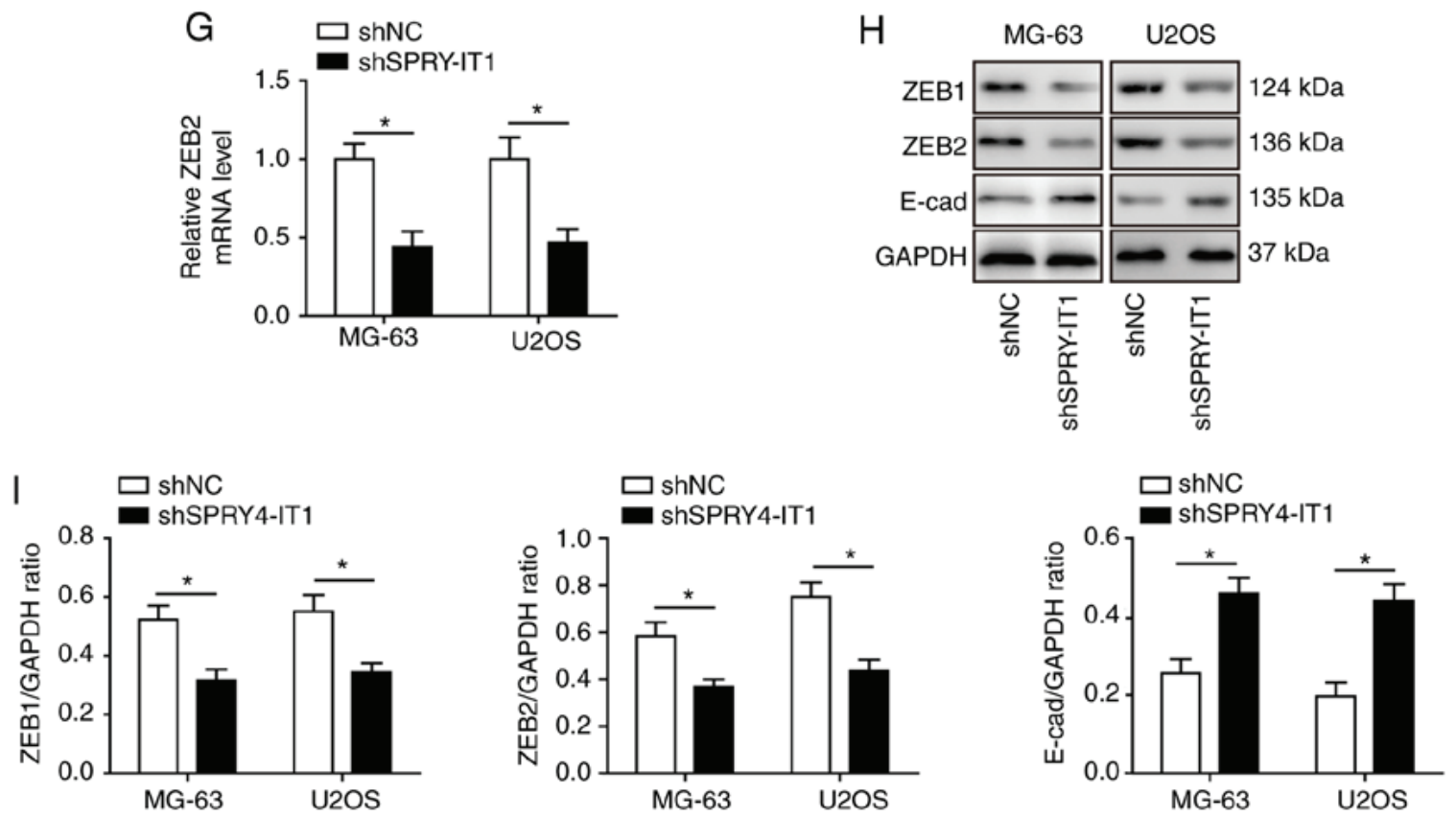

Figure 8. SPRY4-IT1 knockdown exhibits anti-tumour effects in a xenograft mouse model of OS. (A) Xenograft tumours of OS cell lines stably expressing shNC or shSPRY4-IT1. (B) SPRY4-IT1 knockdown significantly reduced tumour volume after 30 days. (C) SPRY4-IT1 knockdown significantly reduced tumour weight in vivo. (D) SPRY4-IT1 levels were significantly lower in the shSPRY4-IT1 xenograft tumour tissues compared with the scrambled control xenograft tumours. (E) miR-101 levels were significantly increased in shSPRY4-IT1 xenograft tumour tissues compared with the scrambled control tumours. (F) ZEB1 mRNA expression levels were significantly lower in the shSPRY4-IT1 xenograft tumour tissues containing shSPRY4-IT1 compared with the scrambled control tumours. (G) ZEB2 mRNA expression levels were significantly lower in shSPRY4-IT1 xenograft tumour tissues compared with the scrambled control tumours. (H and I) ZEB1 and ZEB2 protein expression levels were significantly lower, whereas E-cadherin protein levels were significantly higher, in the shSPRY4-IT1 xenograft tumour tissues compared with the scrambled control tumours. Data are presented as the mean \pm standard deviation of three independent experiments. "P $<0.05,{ }^{* *} \mathrm{P}<0.01$. SPRY4-IT1, sprouty receptor tyrosine kinase signalling antagonist 4-intronic transcript 1; miR, microRNA; OS, osteosarcoma; sh, short hairpin; NC, negative control; ZEB, zinc finger E-box-binding homeoboxes. 
by co-transfection with the miR-101 inhibitor. Together with the dual-luciferase assays showing that ZEB1 and ZEB2 were target genes of miR-101, it was concluded that knockdown of SPRY4-IT1 de-repressed miR-101, leading to the degradation of ZEB1/2. Previously, the expression levels of ZEB1 and ZEB2 were shown to be positively associated with metastatic status in patients with OS $(5,35)$, whereas knockdown of ZEB1 was sufficient to reduce the invasive capacity of OS cells (35). Other published studies also suggested that miR-101 inhibited proliferation, migration and invasion in osteosarcoma cells by targeting ROCK1 or ZEB2 $(36,37)$. However, the present study primarily focused on the SPRY4-IT1/miR-101/ZEB1 or ZEB2 axis in osteosarcoma progression, which have not been reported on by others before, to the best of our knowledge. From a mechanistic perspective, the EMT-modulating functions of ZEBs may contribute to the anti-OS actions of ZEBs (38). For example, it has been demonstrated that ZEB1 can directly bind to the promoter of $\mathrm{CDH} 1$, resulting in the suppression of E-cadherin (product of CDH1) and thus, subsequent induction of EMT (6). In this regard, miR-101 overexpression anticancer actions in vitro may be attributed to reversal of E-cadherin suppression as ZEB1/2 was downregulated.

OS cells which stably expressed SPRY4-IT1 shRNA exhibited significantly lower growth rates in the OS xenograft models. Expression levels of miR-101 were increased in the shSPRY4-IT1 xenograft tumour tissues. Accordingly, the mRNA and protein expression levels of ZEB1 and ZEB2, the target genes of miR-101, were reduced in the SPRY4-IT1 knockdown tumour tissues, and this was accompanied by upregulation of E-cadherin expression in vivo. Therefore, it was concluded that elevated SPRY4-IT1 contributed to the decrease in miR-101 levels in OS cells. The interaction between SPRY4-IT1 and miR-101 then disrupted the inhibition of EMT by upregulating ZEB1 and ZEB2, leading to dysregulated cell growth, migration and invasion.

Although the data in the present study suggested that targeting the SPRY4-IT1/miR-101/ZEBs axis could be a promising approach for the treatment of OS, there were certain limitations. Firstly, despite the data providing evidence that SPRY4-IT1 could interact with miR-101, an RNA pull-down assay, which was not included in the current study, would further strengthen this conclusion. Secondly, the associations between gene expression levels (such as SPRY4-IT1 and miR-101) and the clinical characteristics of patients (such as tumour stage and metastatic status) have not yet been examined, thus, the clinical significance of the dysregulation of this signalling axis is unknown. Future studies on the associations are required and would potentially provide insight into stage-specific therapy. Finally, the mechanism by which SPRY4-IT1 was dysregulated was not investigated in the present study. The upstream regulators of SPRY4-IT1, such as transcription factors, are more likely to be targeted by small molecule chemicals, which are clinically more feasible than shRNA oligos. Future studies on addressing these limitations may demonstrate the clinical significance of SPRY4-IT1/miR-101.

In conclusion, the present study identified a novel SPRY4-IT1/miR-101/ZEBs axis underlying the tumorigenesis of OS, to the best of our knowledge. SPRY4-IT1 may act as a ceRNA to sequester miR-101 in OS as inhibition of SPRY4-IT1 increased function of miR-101, which in turn caused degradation of ZEBs, leading to decreased cell growth, migration and invasion of OS cells. Additionally, the association between SPRY4-IT1 and miR-101 have not been examined in OS previously and the present study is the first to have studied two direct targets (ZEB1 and ZEB2) of miR-101 in OS.

\section{Acknowledgements}

Not applicable.

\section{Funding}

No funding was received.

\section{Availability of data and materials}

The datasets used and/or analysed during the present study are available from the corresponding author on reasonable request.

\section{Authors' contributions}

YH and HG conceived the study. YH, HG, WQY and XWB collected the data. $\mathrm{YH}$ and $\mathrm{HG}$ analysed the data. YH, HG, WQY and XWB performed the experiments. ZHQ and XYC provided the resources and supervised the study. YH, HG, WQY and XWB wrote the original draft. ZHQ and XYC reviewed and edited the manuscript. All authors read and approved the final manuscript.

\section{Ethics approval and consent to participate}

The animal experiments were approved by the Animal Ethics Committee at the Third Affiliated Hospital of Sun Yat-sen University (Guangzhou, China).

\section{Patient consent for publication}

Not applicable.

\section{Competing interests}

The authors declare that they have no competing interests.

\section{References}

1. Anninga JK, Gelderblom H, Fiocco M, Kroep JR, Taminiau AHM, Hogendoorn PC and Egeler RM: Chemotherapeutic adjuvant treatment for osteosarcoma: Where do we stand? Eur J Cancer 47: 2431-2445, 2011.

2. Zhang Y, He Z, Duan Y, Wang C, Kamar S, Shi X, Yang J, Yang J, Zhao N, Han L, et al: Does intensified chemotherapy increase survival outcomes of osteosarcoma patients? A meta-analysis. J bone Oncol 12: 54-60, 2018.

3. Yang G, Yuan J and Li K: EMT transcription factors: Implication in osteosarcoma. Med Oncol 30: 697, 2013.

4. Ye X and Weinberg RA: Epithelial-mesenchymal plasticity: A central regulator of cancer progression. Trends Cell Biol 25: 675-686, 2015.

5. Zhang X and Lei X: Expression of Zeb1 and Zeb2 indicates metastasis and unfavorable prognosis in osteosarcoma. Int J Clin Exp Pathol 10: 611-617, 2017.

6. Eger A, Aigner K, Sonderegger S, Dampier B, Oehler S, Schreiber M, Berx G, Cano A, Beug H and Foisner R: DeltaEF1 is a transcriptional repressor of E-cadherin and regulates epithelial plasticity in breast cancer cells. Oncogene 24: 2375-2385, 2005. 
7. Browne G, Emre Sayan A and Tulchinsky E: ZEB proteins link cell motility with cell cycle control and cell survival in cancer. Cell Cycle 9: 886-891, 2010.

8. Schmitt AM and Chang HY: Long noncoding RNAs in cancer pathways. Cancer Cell 29: 452-463, 2016.

9. Suzuki HI, Young RA and Sharp PA: Super-enhancer-mediated RNA processing revealed by integrative microRNA network analysis. Cell 168: 1000-1014.e15, 2017.

10. Gulino R, Forte S, Parenti R, Memeo L and Gulisano M: MicroRNA and pediatric tumors: Future perspectives. Acta Histochem 117: 339-354, 2015.

11. Fan H, Lu S, Wang S and Zhang S: Identification of critical genes associated with human osteosarcoma metastasis based on integrated gene expression profiling. Mol Med Rep 20: 915-930, 2019.

12. Sampson VB, Yoo S, Kumar A, Vetter NS and Kolb EA MicroRNAs and potential targets in osteosarcoma: Review. Front Pediatr 3: 69, 2015

13. Yang Z, Li X, Yang Y, He Z, Qu X and Zhang Y: Long noncoding RNAs in the progression, metastasis and prognosis of osteosarcoma. Cell Death Dis 7: e2389, 2016.

14. Ergun S and Oztuzcu S: Oncocers: ceRNA-mediated cross-talk by sponging miRNAs in oncogenic pathways. Tumor Biol 36: 3129-3136, 2015.

15. López-Urrutia E, Bustamante Montes LP, Ladrón de Guevara Cervantes D, Pérez-Plasencia C and Campos-Parra AD Crosstalk between long non-coding RNAs, micro-RNAs and mRNAs: Deciphering molecular mechanisms of master regulators in cancer. Front Oncol 9: 669, 2019.

16. Yamamura S, Imai-Sumida $\mathrm{M}$, Tanaka $\mathrm{Y}$ and Dahiya $\mathrm{R}$ : Interaction and cross-talk between non-coding RNAs. Cell Mol Life Sci 75: 467-484, 2018.

17. Karger S, Liu T, He S, Li Z and Dou P: Application of long noncoding RNAs in osteosarcoma: Biomarkers and therapeutic targets. Cell Physiol Biochem 42: 1407-1419, 2017.

18. Li Z, Shen J, Chan MTV and Wu WKK: The long non-coding RNA SPRY4-IT1: An emerging player in tumorigenesis and osteosarcoma. Cell Prolif 51: e12446, 2018.

19. Xu J, Ding R and Xu Y: Effects of long non-coding RNA SPRY4-IT1 on osteosarcoma cell biological behavior. Am J Transl Res 8: 5330-5337, 2016

20. Guo F, Cogdell D, Hu L, Yang D, Sood AK, Xue F and Zhang W: miR-101 suppresses the epithelial-to-mesenchymal transition by targeting ZEB1 and ZEB2 in ovarian carcinoma. Oncol Rep 31: 2021-2028, 2014

21. Liu D, Li Y, Luo G, Xiao X, Tao D, Wu X, Wang M, Huang C, Wang L, Zeng F and Jiang G: LncRNA SPRY4-IT1 sponges miR-101-3p to promote proliferation and metastasis of bladder cancer cells through up-regulating EZH2. Cancer Lett 388: 281-291, 2017.

22. Livak KJ and Schmittgen TD: Analysis of relative gene expression data using real-time quantitative PCR and the 2(-Delta Delta C(T)) method. Methods 25: 402-408, 2001.

23. Hanahan D and Weinberg RA: Hallmarks of cancer: The next generation. Cell 144: 646-674, 2011.
24. Han L, Chen W, Xia Y, Song Y, Zhao Z, Cheng H and Jiang T: miR-101 inhibits the proliferation and metastasis of lung cancer by targeting zinc finger E-box binding homeobox 1. Am J Transl Res 10: 1172-1183, 2018

25. Thomson DW and Dinger ME: Endogenous microRNA sponges: Evidence and controversy. Nat Rev Genet 17: 272-283, 2016.

26. Kalluri R and Weinberg RA: The basics of epithelial-mesenchymal transition. J Clin Invest 119: 1420-1428, 2009.

27. Son $\mathrm{H}$ and Moon A: Epithelial-mesenchymal transition and cell invasion. Toxicol Res 26: 245-252, 2010.

28. Wong TS, Gao W and Chan JY: Transcription regulation of E-cadherin by zinc finger E-box binding homeobox proteins in solid tumors. Biomed Res Int 2014: 921564, 2014.

29. Daw NC, Chou AJ, Jaffe N, Rao BN, Billups CA, Rodriguez-Galindo C, Meyers PA and Huh WW: Recurrent osteosarcoma with a single pulmonary metastasis: A multi-institutional review. Br J Cancer 112: 278-282, 2015.

30. Sánchez Y and Huarte M: Long non-coding RNAs: Challenges for diagnosis and therapies. Nucleic Acid Ther 23 : 15-20, 2013.

31. Faruq O and Vecchione A: microRNA: Diagnostic Perspective. Front Med (Lausanne) 2: 51, 2015

32. Yao ZS, Li C, Liang D, Jiang XB, Tang JJ, Ye LQ, Yuan K, Ren H, Yang ZD, Jin DX, et al: Diagnostic and prognostic implications of serum miR-101 in osteosarcoma. Cancer Biomark 22: 127-133, 2018.

33. Xu Y, Yao Y, Jiang X, Zhong X, Wang Z, Li C, Kang P, Leng K, $\mathrm{Ji} \mathrm{D,} \mathrm{Li} \mathrm{Z,} \mathrm{et} \mathrm{al:} \mathrm{SP1-induced} \mathrm{upregulation} \mathrm{of} \mathrm{lncRNA} \mathrm{SPRY4-IT1}$ exerts oncogenic properties by scaffolding EZH2/LSD1/DNMT1 and sponging miR-101-3p in cholangiocarcinoma. J Exp Clin Cancer Res 37: 81, 2018.

34. Jin J, Chu Z, Ma P, Meng Y and Yang Y: Long non-coding RNA SPRY4-IT1 promotes proliferation and invasion by acting as a ceRNA of miR-101-3p in colorectal cancer cells. Tumor Biol 39: 1010428317716250, 2017

35. Shen A, Zhang Y, Yang H, Xu R and Huang G: Overexpression of ZEB1 relates to metastasis and invasion in osteosarcoma. J Surg Oncol 105: 830-834, 2012.

36. Jiang R, Zhang C, Liu G, Gu R and Wu H: MicroRNA-101 inhibits proliferation, migration and invasion in osteosarcoma cells by targeting ROCK1. Am J Cancer Res 7: 88-97, 2017.

37. Lin H, Zheng X, Lu T, Gu Y, Zheng C and Yan H: The proliferation and invasion of osteosarcoma are inhibited by miR-101 via targetting ZEB2. Biosci Rep 39: BSR20181283, 2019.

38. Zhang P, Sun Y and Ma L: ZEB1: At the crossroads of epithelial-mesenchymal transition, metastasis and therapy resistance. Cell Cycle 14: 481-487, 2015

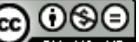

This work is licensed under a Creative Common Attribution-NonCommercial-NoDerivatives 4.0 International (CC BY-NC-ND 4.0) License. 